JOURNAL OF THE

AMERICAN MATHEMATICAL SOCIETY

Volume 11, Number 3, July 1998, Pages 643-667

S 0894-0347(98)00264-1

\title{
A TOPOLOGICAL CHARACTERISATION OF HYPERBOLIC GROUPS
}

\author{
BRIAN H. BOWDITCH
}

\section{INTRODUCTION}

The notion of a hyperbolic group was introduced by Gromov [Gr]. Associated to any hyperbolic group, $\Gamma$, is its boundary, $\partial \Gamma$. This is a metrisable compactum on which $\Gamma$ acts by homeomorphism. If $\Gamma$ is non-elementary (i.e. not finite or virtually cyclic), then $\partial \Gamma$ is perfect. The induced action of $\Gamma$ on the space of distinct triples of $\partial \Gamma$ is properly discontinuous and cocompact. The main result of this paper is that this topological property characterises hyperbolic groups. More precisely:

Theorem 0.1. Suppose that $M$ is a perfect metrisable compactum. Suppose that a group $\Gamma$ acts by homeomorphism on $M$ such that the induced action on the space of distinct triples is properly discontinuous and cocompact. Then, $\Gamma$ is hyperbolic. Moreover, there is a $\Gamma$-equivariant homeomorphism of $M$ onto $\partial \Gamma$.

This appears as a question (No. 3.72), attributed to Gromov, in the problem list compiled by Kirby $[\mathrm{K}]$. It seems that Gromov was already confident that an affirmative answer could be given to this question. The present paper arose from a discussion with Gromov in Melbourne.

We shall also give an alternative characterisation of a hyperbolic group as a convergence group for which every point is a conical limit point (Theorem 8.1). This criterion might be more readily recognised in practice, and also suggests a means of decribing relatively hyperbolic groups dynamically.

We should make a few comments on the hypotheses of Theorem 0.1.

A compactum, $M$, is a compact hausdorff topological space. It is perfect if it has no isolated points. We have assumed that $M$ is metrisable. In fact, this hypothesis can be eliminated (at least in Theorem 0.1), as can be seen by replacing sequences by nets in the arguments throughout this paper. However, we shall not confuse matters by introducing these additional complications here.

Here, we take the space of distinct (ordered) triples, $\Theta_{3}(M)$, of $M$ to be $M \times$ $M \times M$ minus the large diagonal. Clearly, $\Theta_{3}(M)$ is locally compact and hausdorff. The hypothesis that $\Gamma$ acts properly discontinuously on $\Theta_{3}(M)$ is equivalent to the "convergence group" hypothesis of Gehring and Martin [GeM1] (see [GeM2], [Bo4]). In [Bo4], we use the term "uniform convergence group" to describe a group which acts cocompactly as well as properly discontinuously on $\Theta_{3}(M)$.

Convergence groups acting on the circle were analysed by Tukia [T1]. In particular, the results of [T1], [Ga], [CasJ] tell us that a uniform convergence group

Received by the editors March 20, 1997 and, in revised form, February 2, 1998.

1991 Mathematics Subject Classification. Primary 20F32.

(C)1998 American Mathematical Society 
acting on the circle is topologically conjugate to a cocompact Fuchsian group. An important question asks if a uniform convergence group acting on the 2-sphere is conjugate to a cocompact Kleinian group. (See, for example, [CanS] and the references therein for progress on this question.) We remark that a consequence of Theorem 0.1, together with Stallings's theorem on ends and Dunwoody's accessibility result for finitely presented groups [D], is that a uniform convergence group acting on a Cantor set is finitely generated virtually free. (The circle and Cantor set seem to be the only non-trivial examples where uniform convergence groups have been completely classified.)

Among the main ingredients of this paper are notions of a "quasiconformal structure". We shall not attempt a precise definition of this concept. There are several ways of formulating such an idea. Here we shall make use of two, namely "annulus systems" and "crossratios". One of the main objectives is to show how a suitable quasiconformal structure on a set gives rise to a hyperbolic quasimetric on the set of distinct triples. It is well known that, in addition to its topology, the boundary of a hyperbolic group admits a kind of quasiconformal structure. The extent to which such a structure determines the group uniquely (up to quasi-isometry) is explored in $[\mathrm{P}]$. The construction just mentioned can be thought of as addressing the question of existence.

The idea of the proof of Theorem 0.1 is roughly as follows. We use the action of $\Gamma$ to construct an annulus system on $M$. This, in turn, defines a "hyperbolic" crossratio on $M$, which is used to construct a hyperbolic quasimetric on $\Theta_{3}(M)$. From this we deduce that $\Gamma$ is hyperbolic, with $\partial \Gamma$ naturally, and hence $\Gamma$-equivariantly, identified with $M$.

As mentioned earlier, we can, in turn, characterise uniform convergence groups as groups for which every point of $M$ is a conical limit point (Theorem 8.1). In this form, this result has also been obtained by Tukia [T3] using different methods. Together with Theorem 0.1, this gives another characterisation of hyperbolic groups which might be easier to verify in practice. Some of the results of Section 8 (in particular, Proposition 8.2) are valid in a much more general setting. They might be of use, for example, in describing relatively hyperbolic groups dynamically along the lines of [BeM], [Bo2]; see [T3], [Bo5].

The structure of this paper, in outline, is as follows. In Section 1, we introduce some terminology and notation relating to finite metric trees. In Section 2, we define the notion of a "hyperbolic crossratio", and discuss how they are approximated by metric trees. In Section 3, we give a brief review of "quasimetrics" and of hyperbolic (quasi)metric spaces. In Section 4, we show hyperbolic crossratios give rise to hyperbolic quasimetrics (and vice versa). In Section 5, we give another formulation of hyperbolic crossratios. In Section 6, we relate all this to systems of annuli. Finally, in Section 7, we bring convergence actions into the picture, with a view to proving Theorem 0.1. In Section 8, we give another construction of annulus systems and prove Theorem 8.1.

As mentioned earlier, I am indebted to Mikhael Gromov for explaining to me his view of why Theorem 0.1 should be true. The argument I present here is somewhat different, though it was inspired by that discussion. I also thank Craig Hodgson and Walter Neumann for inviting me to the University of Melbourne to take part in the Special Year in geometric group theory. I have also been helped by discussions with Pekka Tukia, particularly in relation to Section 8. I would also like to thank Eric Freden for his interest, and comments on an earlier draft of this paper. 


\section{TREES}

Many of the structures we will be dealing with have a "treelike" nature, so we begin by introducing some notation and terminology relating to trees.

Here a "tree" means a finite simplicial tree, $\tau$. We write $V(\tau)$ for the set of vertices of $\tau$. We write $V_{T}(\tau)$ for the set of terminal vertices (those of degree 1), and write $V_{I}(\tau)=V(\tau) \backslash V_{T}(\tau)$ for the set of internal vertices. For our purposes, we can assume that each internal vertex has degree at least 3. An edge is terminal if one of its endpoints is terminal, otherwise it is internal. Given $x, y \in \tau$, we write $[x, y]$ for the unique arc connecting $x$ and $y$. If $x, y, z \in \tau$, we write $\operatorname{med}(x, y, z)$ for the median of $x, y$ and $z$, i.e. the unique intersection point of the $\operatorname{arcs}[x, y],[y, z]$ and $[z, x]$.

By a metric tree, $(\tau, d)$, we mean a tree, $\tau$, equipped with a path metric, $d$. Such a metric is determined (up to isotopy on the edges) by a map from the set of edges to $(0, \infty)$, which assigns to each edge its rectifiable length (or equivalently the distance between its endpoints).

We note that a metric tree is 0-hyperbolic, i.e. for any $x, y, z, w \in \tau$, we have $d(x, y)+d(z, w) \leq \max \{d(x, z)+d(y, w), d(x, w)+d(y, z)\}$. In fact any finite 0hyperbolic space can be isometrically embedded in a metric tree. The notion of " $k$-hyperbolicity", for a metric space, is defined by relaxing the above inequality by some fixed additive constant (see Section 3).

Given $x, y, z, w \in \tau$, we write

$$
(x y \mid z w)_{\tau}=\frac{1}{2} \max \{0, d(x, z)+d(y, w)-d(x, y)-d(z, w)\} .
$$

In other words, $(x y \mid z w)_{\tau}$ is the distance between the segments $[x, y]$ and $[z, w]$. This operation defines a "crossratio" in that $(x y \mid z w)_{\tau}=(y x \mid z w)_{\tau}=(z w \mid x y)_{\tau}$. (We remark that only the length of the internal edges of $\tau$ are relevant to this definition.) In general a crossratio will be termed "0-hyperbolic" if its restriction to every finite subset arises from a metric tree in this way.

\section{Crossratios}

The main aim of this section is to describe the notion of a hyperbolic crossratio. This is a 4-ary operation defined on a set, with the property that its restriction to any 5-element subset agrees, up to an additive constant, with the crossratio defined on a metric tree, in the manner described in Section 1. It turns out that such 5-element subsets suffice. It automatically follows that any finite subset has the same property, though the additive constant will depend on the cardinality of the set. This is the main result of this section (Theorem 2.1) and is the principal tool for working with such crossratios. It is an exact analogue of the treelike nature of hyperbolic (quasi)metrics (where 4-point sets suffice for the definition). This latter result is well known (see Proposition 3.2).

A notion of crossratio, along similar lines to that described here, has been used by Otal in the case of negatively curved manifolds [O]. It has been observed by several authors that the boundary of a hyperbolic group admits an equivariant crossratio, (.. ...), where $(x y \mid z w)$ could be interpreted, up to an additive constant, as the distance between a bi-infinite geodesic connecting $x$ to $y$ and another connecting $z$ and $w$. We shall shortly explore the formal properties of such a crossratio.

Throughout the rest of this paper, we will adopt the following convention regarding "approximate" inequalities. 
Convention. Given $p, q \in \mathbb{R}$, write $p \simeq_{k} q, p \preceq_{k} q$ and $p \ll_{k} q$ to mean, respectively, $|p-q| \leq k, p \leq q+k$ and $p \leq q-k$. Note that $p \simeq_{k} q \simeq_{k} r$ implies $p \simeq_{2 k} q$, and $p \preceq_{k} q \preceq_{k} r$ implies $p \preceq_{2 k} q$. Since we shall be using many manipulations of this nature, it would be rather tedious to keep track of the precise additive constants involved. As a consequence, we shall frequently drop the subscripts, and behave as though the relations $\simeq$ and $\preceq$ were transitive. At any given point, the constant involved will be some fixed multiple of the initial constant. The factor involved could, in principle, be determined by tracing back through the argument. When we use the phrase "up to an additive constant" it is to be understood that the constant is some fixed multiple of the constant introduced in the hypotheses. We shall sometimes use the dichotomy $p \preceq q$ or $q \ll p$. We shall have no reason to view these as mutually exclusive, or to take the constants to be equal (so long as they exhaust all possibilities). When the dichotomy is introduced, the initial additive constants are assumed to be chosen appropriately for the rest of the argument.

Let $M$ be a set. We write $\Theta_{n}(M)$ for the set of distinct (ordered) $n$-tuples of $M$, i.e. $M^{n}$ minus the large diagonal. By a crossratio we shall mean a map, $[(x, y, z, w) \mapsto(x y \mid z w)]: \Theta_{4}(M) \longrightarrow[0, \infty)$, with the symmetry $(x y \mid z w)=(y x \mid z w)$ $=(z w \mid x y)$ for all $(x, y, z, w) \in \Theta_{4}(M)$.

Definition. We say that a crossratio, (..|..), is $k$-hyperbolic if it satisfies axioms $(\mathrm{C} 1)$ and $(\mathrm{C} 2)$ below.

(C1): If $F \subseteq M$ is a 4-element subset, then we can write $F=\{x, y, z, w\}$ with $(x z \mid y w) \simeq_{k} 0$ and $(x w \mid y z) \simeq_{k} 0$.

(C2): If $F \subseteq M$ is a 5-element subset, then we can write $F=\{x, y, z, w, u\}$ with

$$
\begin{aligned}
(x y \mid z u) & \simeq_{k}(x y \mid w u), \\
(x u \mid z w) & \simeq_{k}(y u \mid z w), \\
(x y \mid z w) & \simeq_{k}(x y \mid z u)+(x u \mid z w),
\end{aligned}
$$

and with $(a b \mid c d) \simeq_{k} 0$ in all other cases where $a, b, c, d \in F$ are distinct (allowing for the symmetries of the crossratio).

We say that (..|..) is hyperbolic if it is $k$-hyperbolic for some $k$.

The conditions $(\mathrm{C} 1)$ and $(\mathrm{C} 2)$ thus tell us respectively that the crossratio restricted to any 4-element or 5-element subset is derived, up to an additive constant, from some metric tree in the manner described in Section 1. In fact, it follows that this is true of all finite subsets:

Theorem 2.1. For all $n \in \mathbb{N}$, there is some constant, $h(n)$, depending only on $n$, such that if (.....) is a $k$-hyperbolic crossratio defined on a set, $F$, of cardinality $n$, then we can embed $F$ in a metric tree, $\tau$, such that for all distinct $x, y, z, w \in F$ we have $\left|(x y \mid z w)-(x y \mid z w)_{\tau}\right| \leq k h(n)$.

We note that we can suppose that, in fact, $F$ is precisely the set, $V_{T}(\tau)$, of terminal vertices of $\tau$. Also, only the lengths of the internal edges of $\tau$ are relevant. We can view the lengths of the terminal edges as being indeterminate.

We also note that the result implies that every 0-hyperbolic crossratio on a finite set arises (precisely) from a metric tree.

Before setting about the proof, we introduce a bit more notation. Given distinct $x, y, z, w \in M$, we shall write $(x y: z w)$ to mean that $(x z \mid y w) \simeq 0$ and $(x w \mid y z) \simeq 0$. In other words, up to an additive constant, the crossratios involving $\{x, y, z, w\}$ arise 


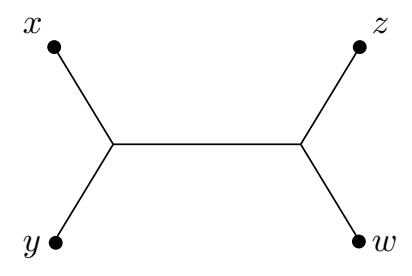

FIGURE 1

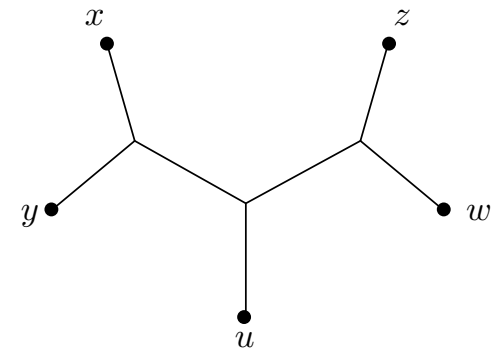

Figure 2

from a finite tree of the combinatorial form in Figure 1. Given distinct $x, y, z, w, u \in$ $M$, we shall write $(x y: u: z w)$ to mean that $(x y: z w),(u y: z w),(x u: z w)$, $(x y: u w)$ and $(x y: z u)$ all hold. In other words, up to an additive constant, the crossratios involving $\{x, y, z, w, u\}$ arise from a finite tree of the form in Figure 2.

We make a few observations which follow easily from axioms (C1) and (C2). Firstly, given that any $x, y, z, w, u \in M$ are all distinct, we have $(x y \mid z w) \preceq(x u \mid z w)+$ $(x y \mid z u)$. Secondly, if $x, y, z, w \in M$ and $(x y \mid z w) \gg 0$, then $(x y: z w)$. Thirdly, if $x, y, z, w, u \in M$ with $(x u \mid z w) \gg 0$ and $(x y \mid z u) \gg 0$, then $(x y: u: z w)$.

We now set about the proof of Theorem 2.1.

Lemma 2.2. Suppose that (..|..) is a hyperbolic crossratio defined on a finite set $F$. Then, we can find distinct points, $a, b \in F$, such that for any distinct $x, y \in F \backslash\{a, b\}$ we have $(a b: x y)$.

(Here the additive constant does not depend on the cardinality of $F$.)

Proof. Choose distinct $a, b, c, d \in F$ so that $(a b \mid c d)$ is maximal among all values of the crossratio on $F$. If $(a b \mid c d) \simeq 0$, there is nothing to prove, so we can suppose that $(a b \mid c d) \gg 0$.

We claim that, for all $x \in F$, we have $(a x \mid b c) \simeq 0$. For if $(a x \mid b c) \gg 0$, then we have $(a x: b: c d)$, and so $(a x \mid c d) \simeq(a x \mid b c)+(a b \mid c d)$. Thus $(a x \mid c d)>(a b \mid c d)$, contradicting the maximality of $(a b \mid c d)$. This proves the claim. Similarly, we have $(a c \mid b y) \simeq 0$ for all $y \in F$.

Now, suppose $x, y \in F$. We get $(a x \mid b y) \preceq(a c \mid b y)+(a x \mid b c) \simeq 0+0=0$. Thus, for all $x, y \in F$, we have $(a x \mid b y) \simeq 0$ and $(a y \mid b x) \simeq 0$, and so $(a b: x y)$ holds as claimed.

It follows that if $x, y, z \in F \backslash\{a, b\}$ are distinct, then one of $(a b: x: y z)$, $(a b: y: z x)$ or $(a b: z: x y)$ must hold. By considering all three possibilities, we see easily that:

Lemma 2.3. If $F, a, b$ are as in Lemma 2.2, and $x, y, z \in F \backslash\{x, y\}$ are distinct, then $(a x \mid y z) \simeq(b x \mid y z)$ and $(a b \mid x z)+(b z \mid x y) \simeq(a b \mid x y)+(b y \mid x z)$.

Proof of Theorem 2.1. Suppose, by induction, that Theorem 2.1 holds for all sets of cardinality $n$. Suppose that $F$ has cardinality $n+1$. Choose $a, b \in F$ as given by Lemma 2.2, and let $P=F \backslash\{a\}$. We embed $P$ in a metric tree, $\sigma$, as given by the inductive hypothesis. We thus have $(x y \mid z w) \simeq(x y \mid z w)_{\sigma}$ for all distinct $x, y, z, w \in P$. Here, the additive constant defining the relation $\simeq$ depends on $n$ as well as the hyperbolicity constant. We can assume that $P=V_{T}(\sigma)$. We shall also assume that all interior vertices of $\sigma$ have degree at least 3 . 
Let $v \in V_{I}(\sigma)$ be the vertex adjacent to the vertex $b$. Thus, the $\operatorname{arc}[b, v]$ is a terminal edge. Choose a point $v^{\prime}$ in the interior of this edge. We subdivide $[b, v]$ into two edges $\left[b, v^{\prime}\right]$ and $\left[v^{\prime}, v\right]$, and connect $a$ to $v^{\prime}$ by adjoining another terminal edge, $\left[a, v^{\prime}\right]$. This gives us a new tree, $\tau=\sigma \cup\left[a, v^{\prime}\right]$, with $F=V_{T}(\tau)$.

So far, we have not assigned any length to the new internal edge $\left[v, v^{\prime}\right]$. To this end, choose points $c, d \in P \backslash\{b\}$ in different components of $\sigma \backslash\{v\}$, so that $v \in[c, d]$. It follows that, in $\tau,\left[v, v^{\prime}\right]=[a, c] \cap[b, d]$. We now assign a length of $(a b \mid c d)$ to $\left[v, v^{\prime}\right]$. Thus, $\tau$ is a metric tree (with the terminal edges of indeterminate length). From the construction, $(a b \mid c d)_{\tau}=(a b \mid c d)$. (If $(a b \mid c d)$ happens to be 0 , we can take $v=v^{\prime}$.) We note that if $x, y, z, w \in F \backslash\{a\}$, then $(w x \mid y z)_{\tau}=(w x \mid y z)_{\sigma} \simeq(w x \mid y z)$, in particular we note that $(b x \mid y z)_{\tau} \simeq(b x \mid y z)$. It remains to consider the other crossratios.

Now, if $x, y \in F \backslash\{a, b\}$ we see, from the construction, that $[a, b] \cap[x, y]=$ $\emptyset$. Thus if $x, y, z \in F \backslash\{a, b\}$ are distinct, the combinatorial possibilities for the subtree of $\tau$ spanned by $\{a, b, x, y, z\}$ are exactly the same as those considered in Lemma 2.3. Therefore, the same argument yields $(a x \mid y z)_{\tau}=(b x \mid y z)_{\tau}$ and $(a b \mid x z)_{\tau}+(b z \mid x y)_{\tau}=(a b \mid x y)_{\tau}+(b y \mid x z)_{\tau}$. We see immediately, using Lemma 2.3, that $(a x \mid y z) \simeq(b x \mid y z) \simeq(b x \mid y z)_{\tau}=(a x \mid y z)_{\tau}$.

It remains to consider crossratios of the form $(a b \mid x y)$. We first note that if $x, y, z \in F \backslash\{a, b\}$ are distinct, then $(a b \mid x y) \simeq(a b \mid x y)_{\tau}$ implies $(a b \mid x z) \simeq(a b \mid x z)_{\tau}$. This follows from the quasi-identities $(b z \mid x y) \simeq(b z \mid x y)_{\tau}$ and $(b y \mid x z) \simeq(b y \mid x z)_{\tau}$, together with the second quasi-identity of Lemma 2.3 , and the corresponding identity in $\tau$ stated above.

Applying the above observation twice, we see that for all distinct $x, y \in F \backslash\{a, b\}$, we have $(a b \mid c d)=(a b \mid c d)_{\tau}$ and so $(a b \mid x d) \simeq(a b \mid x d)_{\tau}$ and so $(a b \mid x y) \simeq(a b \mid x y)_{\tau}$.

This deals with all possible crossratios arising on $F$, and so proves the result. $F$.

We refer to the tree, $\tau$, arising in Theorem 2.1 as an "approximating tree" for

We next want to consider how a crossratio gives rise to a natural topology.

Suppose that (..|..) is a crossratio on a set $M$. Suppose $a, b \in M$ are distinct points. We first define a topology on $M \backslash\{a, b\}$. Given $x \in M \backslash\{a, b\}$ and $r \geq 0$, let $D_{a b}(x, r)=\{x\} \cup\{y \in M \backslash\{a, b, x\} \mid(a b \mid x y) \geq r\}$. We take as base of neighbourhoods for $x$ the collection $\left\{D_{a b}(x, r) \mid r>0\right\}$. We note that if we take some constant $\lambda>1$ sufficiently close to 1 in relation to the hyperbolicity constant, then the map $\left[(x, y) \mapsto \lambda^{-(a b \mid x y)}\right]$ defines a "quasi-ultrametric" which in turns gives us a metric, by the process described in $[\mathrm{GhH}]$. This process changes distance by at most a multiplicative constant, and so it's easily seen that the construction described above does indeed define a neighbourhood base for a metrisable topology.

We claim that if $a, b, c \in M$ are distinct, then the topologies thus defined on $M \backslash\{a, b\}$ and $M \backslash\{a, c\}$ agree. This follows from the inequalities $(a c \mid x y) \leq$ $(a b \mid x y)+p$ and $(a b \mid x y) \leq(a c \mid x y)+q$, where $p=(a c \mid b x)$ and $q=(a b \mid c x)$ are fixed. In other words, $D_{a c}(x, r) \subseteq D_{a b}(x, r-p)$ and $D_{a b}(x, r) \subseteq D_{a c}(x, r-q)$. This proves the claim.

Applying this observation twice, we see that if $a, b, c, d \in M$, with $a \neq b$ and $c \neq d$, then the topologies on $M \backslash\{a, b\}$ and $M \backslash\{c, d\}$ agree on $M \backslash\{a, b, c, d\}$. We thus get a well defined topology on $M$. Clearly, this topology is hausdorff. It's also easy to see from the earlier remarks that it is metrisable. We refer to the topology thus defined on $M$ as the crossratio topology. 
Definition. We say that a crossratio is perfect if the crossratio topology is perfect.

In other words, given any distinct, $x, y, z \in M$, we can find a sequence $\left(x_{i}\right)_{i \in \mathbb{N}}$ in $M \backslash\{x, y, z\}$ with $\left(x x_{i} \mid y z\right) \rightarrow \infty$.

We make another definition which is central to later applications.

Definition. A hyperbolic crossratio is a path crossratio if, given any distinct $x, y, z, w \in M$ and any $p \leq(x y \mid z w)$, there is some $u \in M$ with $(x y: u: z w)$ and with $(x y \mid z u) \simeq p$.

This definition is relative to some additive constant, which we can assume to be equal to the constant of hyperbolicity (by increasing the latter if necessary).

Given the hyperbolicity assumption, it's not hard to see that the path hypothesis is equivalent to the following assertion. Given any distinct $x, y, z, w \in M$, there is a finite sequence of points, $u_{0}, u_{1}, \ldots, u_{n}$ of $M$, with $u_{0}=y, u_{n}=w$, and with $\left(x u_{i} \mid y u_{j}\right) \simeq j-i$ for all $i, j \in\{1, \ldots, n\}$ with $i<j$. (It would be more natural to take $\left(x u_{i} \mid y u_{j}\right) \simeq K(j-i)$ for some constant, $K$, related to the hyperbolicity constant. In this way, we could insist, for example, that $\left(x u_{i}: y u_{j}\right)$. We shall not need this. In any case, we can always scale so that $K=1$, to save burdening our notation.)

We note:

Lemma 2.8. Suppose (..|..) is a perfect hyperbolic path crossratio on a set $M$. Suppose that $a, b, c \in M$ are distinct. Then, there is a bi-infinite sequence, $\left(x_{i}\right)_{i \in \mathbb{Z} \text {, }}$ of points of $M \backslash\{a, b\}$ such that $\left(b x_{i} \mid a x_{j}\right) \simeq j-i$ for all $i, j \in \mathbb{Z}$, and with $x_{0}=c$.

Proof. By perfection, we can find a bi-infinite sequence $\left(y_{i}\right)_{i}$ with $y_{i} \rightarrow a$ and $y_{-i} \rightarrow b$, and with $y_{0}=c$. Passing to a subsequence (retaining $y_{0}$ ), we can suppose that if $0 \leq i<j$, then $\left(b y_{0} \mid a y_{i}\right) \ll\left(b y_{0} \mid a y_{j}\right)$ and $\left(a y_{0} \mid b y_{-i}\right) \ll\left(a y_{0} \mid b y_{-j}\right)$. It follows easily that for any $i<j$, we have $\left(b y_{i} \mid a y_{j}\right) \gg 0$. We can now use the path property to interpolate between these points.

Note that for such a sequence, we necessarily have $x_{i} \rightarrow a$ and $x_{-i} \rightarrow b$ as $i \rightarrow \infty$.

\section{QuAsimetrics}

A quasimetric is a metric, except that we relax the triangle inequalities by an additive constant. On a large scale, a quasimetric is indistinguishable from a metric, so all the standard results about quasi-isometries and hyperbolicity etc. go through, more or less unchanged. What we lose is the notion of an induced topology, so we shall have to find geometric substitutes for ideas of local compactness etc. Here is a precise definition:

Definition. A k-quasimetric, $\rho$, on a set, $Q$, is a function $\rho: Q^{2} \longrightarrow[0, \infty)$ satisfying $\rho(x, x)=0, \rho(x, y)=\rho(y, x)$ and $\rho(x, y) \leq \rho(x, z)+\rho(z, y)+k$ for all $x, y, z \in Q$.

A quasimetric is a $k$-quasimetric for some $k \geq 0$. We refer to $k$ as the "quasimetric constant". (Note that a 0 -quasimetric is the same as a pseudometric.) Given $x \in Q$ and $r \geq 0$, write $N_{\rho}(x, r)=\{y \in Q \mid \rho(x, y) \leq r\}$. Given $P \subseteq Q$, write $N_{\rho}(P, r)=\bigcup_{x \in P} N_{\rho}(x, r)$. We say that $P$ is $r$-quasidense in $Q$ if $Q=N_{\rho}(P, r)$.

A $k$-geodesic segment (connecting $x_{0}$ to $x_{n}$ ) is a finite sequence of points $x_{0}, x_{1}, \ldots, x_{n}$ with $\rho\left(x_{i}, x_{j}\right) \simeq_{k}|i-j|$ for all $i, j \in\{1, \ldots, n\}$. 
Definition. We say that a quasimetric is a path quasimetric if there is some $k \geq 0$ such that every pair of points can be connected by a $k$-geodesic segment.

We may as well assume that $k$ is also the quasimetric constant.

We similarly define the notions of geodesic ray and bi-infinite geodesic, allowing infinite and bi-infinite sequences (with domains $\mathbb{N}$ and $\mathbb{Z}$ respectively).

We define the notion of quasi-isometry between quasimetric spaces, $Q$ and $Q^{\prime}$, exactly as for metric spaces - namely a map $f: Q \longrightarrow Q^{\prime}$ which distorts distances by at most a linearly bounded amount, and such that $f(Q)$ is quasidense in $Q^{\prime}$. The property of being a path quasimetric is thus quasi-isometry invariant. Note also that if $P \subseteq Q$ is quasidense, then the inclusion of $(P, \rho)$ in $(Q, \rho)$ is a quasi-isometry.

It turns out that every path quasimetric space is quasi-isometric to a graph (and hence to a genuine path metric space) as can be seen from the following construction.

Suppose that $(Q, \rho)$ is a path quasimetric, and $r \geq 0$. Let $G=G_{r}(Q)$ be the graph with vertex set $Q$ defined by connecting two vertices $x, y \in Q$ by an edge if $\rho(x, y) \leq r$. We put a path-metric, $\omega$, on $G$ by deeming every edge to have unit length. Provided we take $r$ to be sufficiently large in relation to the quasimetric constant, we see easily that $G$ is connected (so that $\omega$ is indeed a metric) and that the inclusion of $(Q, \rho)$ in $(G, \omega)$ is a quasi-isometry.

In this generality, this result is of little interest to us, though it may be somewhat reassuring.

Definition. A quasimetric space is locally finite if every bounded subset is finite.

Thus, if $(Q, \rho)$ is a locally finite path quasimetric space, then the graph constructed above will be locally finite (in the sense that every vertex has finite degree).

For future reference, we note:

Lemma 3.1. Suppose that a group, $\Gamma$, admits a left invariant, locally finite, path quasimetric, $\rho$. Then, $\gamma$ is finitely generated. Moreover, $(\Gamma, \rho)$ is quasi-isometric (via the identity) to $\Gamma$ in the word metric with respect to some (and hence any) finite generating set.

Proof. In this case, the graph $G_{r}(\Gamma)$ is just the Cayley graph of $\Gamma$ defined for the finite set $\Gamma_{0}=N_{\rho}(e, r)$ where $e$ is the identity. Since $G_{r}(\Gamma)$ is connected, we see that $\Gamma_{0}$ generates $\Gamma$.

Towards the end of this section, we will consider the general question of when a path quasimetric space is quasi-isometric to a locally finite space. We do not formally need to worry about this for the purposes of this paper, though it helps to clarify a few points arising out of the construction. First, we get on with more important things, like crossratios and hyperbolicity.

Suppose that $(Q, \rho)$ is a quasimetric space (not necessarily a path quasimetric for the moment). Given $x, y, z, w \in Q$, set

$$
\begin{array}{r}
(x y \mid z w)_{\rho}=(1 / 2)(\max \{\rho(x, y)+\rho(z, w), \rho(x, z)+\rho(y, w), \rho(x, w)+\rho(y, z)\} \\
-(\rho(x, y)+\rho(z, w))) .
\end{array}
$$

This has the symmetry $(x y \mid z w)_{\rho}=(y x \mid z w)_{\rho}=(z w \mid x y)_{\rho}$ and its restriction to $\Theta_{4}(Q)$ is a crossratio on $Q$. We can express hyperbolicity in these terms:

Definition. We say that a quasimetric space, $(Q, \rho)$, is hyperbolic if the induced crossratio, $(. . \mid . .)_{\rho}$, on $Q$ satisfies axiom (C1) of a hyperbolic crossratio. 
We refer to the constant involved as the hyperbolicity constant, which we may as well take to be equal to the quasimetric constant. In the case of metric, this is precisely the standard "four-point" characterisation of hyperbolicity.

The notion of a hyperbolic metric space was defined by Gromov [Gr]. For further elaboration, see, for example, $[\mathrm{GhH}]$ or [Bo1]. We freely use arguments or results from these papers, where they generalise, without any essential change, to quasimetrics. For example, the following analogue of Theorem 2.1 can be found (for metrics) in any of the above references:

Proposition 3.2. For all $n \in \mathbb{N}$, there is some constant, $h(n)$, depending only on $n$, such that if $(F, \rho)$ is a k-hyperbolic $k$-quasimetric space of cardinality $n$, then we can embed $F$ in a metric tree, $(\tau, d)$, such that $|\rho(x, y)-d(x, y)| \leq k$ for all $x, y \in F$.

(Note that the definition of hyperbolicity amounts to the conclusion of Proposition 3.2 for all 4 -element subsets.)

Now, suppose that $(Q, \rho)$ is hyperbolic. Applying Proposition 3.2 to every 5element subset of $Q$, we see easily that (..|..) is a hyperbolic crossratio. (In other words, axiom $(\mathrm{C} 1)$ automatically implies axiom $(\mathrm{C} 2)$ in the case where the crossratio is derived from a quasimetric.)

We note that a hyperbolic quasimetric is a path quasimetric if and only if the following holds. If $x, y \in Q$ and $p \leq \rho(x, y)$, then there is some $z \in Q$ with $\rho(x, z) \simeq p$ and $\rho(y, z) \simeq \rho(x, y)-p$.

Suppose that $(Q, \rho)$ is a hyperbolic path quasimetric space. Given any three points, $x, y, z \in Q$, we can find a centre for $(x, y, z)$, namely a point $w \in Q$ such that $(x y \mid w w)_{\rho} \simeq 0,(y z \mid w w)_{\rho} \simeq 0$ and $(z x \mid w w)_{\rho} \simeq 0$. Moreover, if $w^{\prime}$ is another centre of $(x, y, z)$, then $\rho\left(w, w^{\prime}\right) \simeq 0$.

We have the standard definition of the boundary, $\partial Q$, of $Q$. To describe this, fix a basepoint, $a \in Q$, and let $\partial Q$ be the set of parallel classes of geodesic rays emanating from $a$. (Two rays are "parallel" if they remain a bounded distance apart.) Fix some $r \gg 0$. Given $x \in \partial Q$, choose a ray, $\left(x_{i}\right)_{i}$, in the class of $x$. Given $n \in \mathbb{N}$, let $D(n)$ be the set of all $y \in Q \cup \partial Q$ such that some geodesic connecting $a$ to $y$ (i.e. a ray in the class of $y$, if $y \in \partial Q$ ) meets $N_{\rho}\left(x_{n}, r\right)$. The collection $\{D(n) \mid n \in \mathbb{N}\}$ defines a neighbourhood base of $x$ in $Q \cup \partial Q$. For definiteness, we take the topology on $Q$ to be discrete. We routinely check, as in the metric case, that this all works to give us a well defined topology (independent of the various choices) on $Q \cup \partial Q$. In fact, this topology is metrisable.

Suppose, now, that $\left(x_{1}, x_{2}, x_{3}, x_{4}\right) \in \Theta_{4}(\partial Q)$. We find pairwise disjoint open subsets, $O_{1}, O_{2}, O_{3}, O_{4}$, of $Q \cup \partial Q$, with $x_{i} \in O_{i}$, such that if $y_{i}, z_{i} \in Q \cap O_{i}$, then $\left(y_{1} y_{2} \mid y_{3} y_{4}\right)_{\rho} \simeq\left(z_{1} z_{2} \mid z_{3} z_{4}\right)_{\rho}$. Let $\left(O_{1} O_{2} \mid O_{3} O_{4}\right)_{\rho}$ be the supremum of $\left(y_{1} y_{2} \mid y_{3} y_{4}\right)_{\rho}$ as each $y_{i}$ varies over $Q \cap O_{i}$. Let $\left(x_{1} x_{2} \mid x_{3} x_{4}\right)_{\rho}$ be the limit of $\left(O_{1} O_{2} \mid O_{3} O_{4}\right)_{\rho}$ as each set $O_{i}$ shrinks to the point $x_{i}$. We see easily that this defines a hyperbolic crossratio, $(. . \mid . .)_{\rho}$, on $\partial Q$. Moreover, we see that the topology on $\partial Q$ agrees with the crossratio topology.

In fact, we can hold fixed any of the points in the above construction. In this way we get natural definitions of $(a b \mid c x)_{\rho},(a b \mid x y)_{\rho}$ and $(a x \mid y z)_{\rho}$, where $a, b, c \in Q$ and $x, y, z$ are distinct points of $\partial Q$. (Indeed, we get a hyperbolic crossratio on $Q \cup \partial Q$.) Note that, if $(x, y, z) \in \Theta_{3}(\partial Q)$, then we can define a "centre" of $(x, y, z)$ as a point, $w \in Q$, for which $(x y \mid w w)_{\rho} \simeq 0,(y z \mid w w)_{\rho} \simeq 0$ and $(z x \mid w w)_{\rho} \simeq 0$. 
As with path metric spaces, the property of being hyperbolic is quasi-isometry invariant. In fact, a quasi-isometry $f: Q \longrightarrow Q^{\prime}$ of hyperbolic path quasimetric spaces gives rise naturally to a homeomorphism of $\partial Q$ to $\partial Q^{\prime}$.

Using this observation, together with Lemma 3.1, we note:

Lemma 3.3. Suppose that $\Gamma$ admits a left invariant, locally finite, hyperbolic path quasimetric, $\rho$. Then $\Gamma$ is hyperbolic. Moreover, the Gromov boundary, $\partial \Gamma$, is naturally (and hence $\Gamma$-equivariantly) homeomorphic to the boundary of $(\Gamma, \rho)$.

The rest of this section is not formally needed for the main result of this paper, but helps to clarify a couple of points arising.

Note that the boundary of a locally finite hyperbolic path quasimetric space is necessarily compact. This is therefore also true of a quasimetric space quasiisometric to such. It turns out there is a kind of converse to this observation (Proposition 3.5).

Let us say that a path quasimetric is almost locally finite if it is quasi-isometric to a locally finite quasimetric. We first aim to give an alternative formulation of this. One observation to make is that the image of a locally finite quasimetric under a quasi-isometry is, itself, locally finite. Thus, a quasimetric is almost locally finite if and only if it contains a locally finite quasidense subset. Here is another criterion:

We say that a quasimetric space, $(P, \rho)$, is $r$-separated if $\rho(x, y) \geq r$ for all distinct $x, y \in P$.

Lemma 3.4. A path quasimetric space, $(Q, \rho)$, is almost locally finite if and only if there is some $r \geq 0$ such that every bounded $r$-separated subset of $Q$ is finite.

Proof. Clearly, every locally finite space has this property for any $r \geq 0$. Also this property is quasi-isometry invariant. (The image of an $r$-separated subset under a quasi-isometry is $(r-k)$-separated, where $k$ is a constant depending on the quasiisometry constants. Boundedness is also preserved.)

Conversely, if the property holds, let $P \subseteq Q$ be a maximal $r$-separated subset. Thus, $P$ is locally finite. Moreover $P$ is $(2 r)$-quasidense in $Q$.

Proposition 3.5. Suppose that $(Q, \rho)$ is a hyperbolic path quasimetric space, whose boundary, $\partial Q$, is compact. Suppose that every point of $Q$ lies a bounded distance from the centre of some triple in $\Theta_{3}(\partial Q)$. Then, $Q$ is almost locally finite.

Proof. Choose $r>0$, large in relation to the hyperbolicity constant and the bound in the hypothesis. If the criterion of Lemma 3.4 fails, then we can find an infinite bounded sequence, $\left(w_{i}\right)$, of points of $Q$ such that $\rho\left(w_{i}, w_{j}\right) \geq r$ whenever $i \neq$ $j$. Now, $w_{i}$ is a bounded distance from the centre of a triple of distinct points, $x_{i}, y_{i}, z_{i} \in \partial Q$. Passing to a subsequence, we can suppose that $x_{i} \rightarrow x, y_{i} \rightarrow y$ and $z_{i} \rightarrow z$. Since the set of $w_{i}$ is bounded, it follows easily that $x, y$ and $z$ are all distinct. Let $w \in Q$ be a centre for $(x, y, z)$. Now, for large $i, \rho\left(w, w_{i}\right)$ is bounded by some constant depending on some a priori constant which we can assume is less than $r / 2$. This contradicts the fact that $\rho\left(w_{i}, w_{j}\right) \geq r$ for $i \neq j$.

\section{Crossratios to quasimetrics}

In this section, we describe how a hyperbolic crossratio on a set gives rise to a hyperbolic quasimetric on the set of distinct triples. The key to this will be the use of approximating trees for crossratios as given by Theorem 2.1. 
Let (.....) be a hyperbolic crossratio on a set, $M$. It will be convenient to adopt the natural convention that $(x y \mid x y)=(x y \mid x z)=0$ for all distinct $x, y, z \in M$. Let $Q=\Theta_{3}(M)$ be the set of distinct triples of $M$. Given two elements, $X=\left(x_{1}, x_{2}, x_{3}\right)$ and $Y=\left(y_{1}, y_{2}, y_{3}\right)$, of $M$, we define $\rho(X, Y) \in[0, \infty)$ as follows. Let

$$
\rho(X, Y)=\max \left\{\left(x_{i} x_{j} \mid y_{k} y_{l}\right) \mid i, j, k, l \in\{1,2,3\}, i \neq j, k \neq l\right\} .
$$

We thus have $\rho(X, X)=0$ and $\rho(X, Y)=\rho(Y, X)$ for all $X, Y \in Q$. (In fact, we note that $\rho(X, Y)=0$ if $\left\{x_{1}, x_{2}, x_{3}\right\}=\left\{y_{1}, y_{2}, y_{3}\right\}$.)

A more geometric interpretation of this definition is as follows. Choose an approximating tree, $(\tau, d)$, for the set $\left\{x_{1}, x_{2}, x_{3}, y_{1}, y_{2}, y_{3}\right\}$ (as given by Theorem 2.1). Let $x$ and $y$ be, respectively, the medians in $\tau$ of the triples $X$ and $Y$ (i.e. $x=\operatorname{med}\left(x_{1}, x_{2}, x_{3}\right)$ and $\left.y=\operatorname{med}\left(y_{1}, y_{2}, y_{3}\right)\right)$. Then:

Lemma 4.1. $\rho(X, Y) \simeq d(x, y)$.

Proof. Note that if $i, j, k, l \in\{1,2,3\}$ with $i \neq j$ and $k \neq l$, then the segments $\left[x_{i}, x_{j}\right]$ and $\left[y_{k}, y_{l}\right]$ contain, respectively, the points $x$ and $y$. Thus, $\left(x_{i} x_{j} \mid y_{k} y_{l}\right) \simeq$ $\left(x_{i} x_{j} \mid y_{k} y_{l}\right)_{\tau} \leq d(x, y)$. It follows that $\rho(X, Y) \preceq d(x, y)$.

Conversely, we can find $i, j$ so that $x_{i}, x_{j}$ and $y$ lie in different components of $\tau \backslash\{x\}$; in other words, $x=\operatorname{med}\left(x_{i}, x_{j}, y\right)$. We can similarly find $k, l$ so that $y=\operatorname{med}\left(y_{k}, y_{l}, x\right)$. Thus, $d(x, y)=\left(x_{i} x_{j} \mid y_{k} y_{l}\right)_{\tau} \simeq\left(x_{i} x_{j} \mid y_{k} y_{l}\right)$, and so $d(x, y) \preceq$ $\rho(X, Y)$.

Proposition 4.2. $(Q, \rho)$ is a hyperbolic quasimetric space.

Proof. To see that $\rho$ is a quasimetric, consider three points, $X=\left(x_{1}, x_{1}, x_{3}\right)$, $Y=\left(y_{1}, y_{2}, y_{3}\right)$ and $Z=\left(z_{1}, z_{2}, z_{3}\right)$, in $Q$. Let $(\tau, d)$ be an approximating tree for the set $\left\{x_{1}, x_{2}, x_{3}, y_{1}, y_{2}, y_{3}, z_{1}, z_{2}, z_{3}\right\}$. Let $x, y, z \in \tau$ be, respectively, the medians of the triples, $X, Y$ and $Z$. By Lemma 4.1, we have $\rho(X, Y) \simeq d(x, y)$, $\rho(Y, Z) \simeq d(y, z)$ and $\rho(Z, X) \simeq d(z, x)$. Now, $d(x, y) \leq d(x, z)+d(z, y)$ and so $\rho(X, Y) \simeq \rho(X, Z)+\rho(Z, Y)$.

The proof that $\rho$ is hyperbolic is similar. We consider a fourth point, $W=$ $\left(w_{1}, w_{2}, w_{3}\right)$, and let $(\tau, d)$ be an approximating tree for the set $\bigcup_{i=1}^{3}\left\{x_{i}, y_{i}, z_{i}, w_{i}\right\}$. The result now follows from the fact that $(\tau, d)$ is 0-hyperbolic, using the "fourpoint" characterisation of hyperbolicity, as described in Section 3.

Lemma 4.3. If (..|..) is a path crossratio, then $\rho$ is a path quasimetric.

Proof. Suppose $X=\left(x_{1}, x_{2}, x_{3}\right)$ and $Y=\left(y_{1}, y_{2}, y_{3}\right)$. Without loss of generality, we can suppose that $\rho(X, Y)=\left(x_{1} x_{2} \mid y_{1} y_{2}\right)$, and that $\rho(X, Y) \gg 0$. Given any $p \leq$ $\rho(X, Y)$, we have some $u \in M$ such that $\left(x_{1} x_{2}: u: y_{1} y_{2}\right)$, and with $\left(x_{1} x_{2} \mid y_{1} u\right) \simeq p$. It follows that $\left(x_{1} u \mid y_{1} y_{2}\right) \simeq \rho(Y, Z)-p$. In the case of interest, we can suppose that $0 \ll p \ll \rho(X, Y)$. Let $Z=\left(x_{1}, y_{1}, u\right)$. Let $(\tau, d)$ is an approximating tree for $\left\{x_{1}, x_{2}, x_{3}, y_{1}, y_{2}, y_{3}, u\right\}$, and let $x, y, z \in \tau$ be the medians, respectively, of $X, Y$ and $Z$. Thus, $z \in[x, y]$. We see that $\rho(X, Z) \simeq d(x, z) \simeq p$ and $\rho(Y, Z) \simeq d(y, z) \simeq$ $\rho(X, Y)-p$, as required.

It follows that $Q$ has a well defined boundary, $\partial Q$, as described in Section 3, which itself admits a crossratio $(. . \mid . .)_{\rho}$ induced by the quasimetric $\rho$. In the case where $\rho$ is perfect, we aim to construct a natural embedding of $M$ in $\partial Q$, respecting this crossratio up to an additive constant. Specifically we shall show: 
Proposition 4.4. Suppose that (..|..) is a perfect hyperbolic path crossratio on a set, $M$. Let $\rho$ be the hyperbolic path quasimetric defined on the set, $Q$, of distinct triples, as described above. Then there is a natural embedding of $M$ in the boundary, $\partial Q$, such that for all $x, y, z, w \in M$, we have $(x y \mid z w) \simeq(x y \mid z w)_{\rho}$. Moreover, $M$ is dense in $\partial Q$.

Recall that the standard topology on $\partial Q$ agrees with the crossratio topology induced by the crossratio $(. . \mid . .)_{\rho}$. It follows that the subspace topology on $M$ agrees with the topology induced by the crossratio (.....) (so the embedding of $M$ in $\partial Q$ is necessarily continuous).

The procedure for constructing the embedding is fairly obvious. Suppose that $a, b \in M$ and that $\left(x_{i}\right)_{i \in \mathbb{N}}$ is a sequence of points of $M \backslash\{a, b\}$. We refer to $\left(b,\left(x_{i}\right)\right)$ as a boundary ray tending to $a$ if $\left(b x_{i} \mid a x_{j}\right) \simeq j-i$ whenever $i<j$. Let $X_{i}=\left(b, a, x_{i}\right)$. It follows that, if $i, j \in \mathbb{N}$, then $\rho\left(X_{i}, X_{j}\right) \simeq|i-j|$. (Note that if $x, y, z, w \in M$ are distinct, then $\rho((x, y, z),(x, y, w)) \simeq \max \{(x z \mid y w),(x w \mid y z)\}$.) In other words, $\left(X_{i}\right)_{i \in \mathbb{N}}$ is a geodesic ray in $(Q, \rho)$. Note that, by Lemma 2.8 , such rays exist for any $a \in M$.

Lemma 4.5. Suppose that $\left(b,\left(x_{i}\right)\right)$ and $\left(c,\left(y_{i}\right)\right)$ are boundary rays tending to $a \in$ $M$. Let $X_{i}=\left(b, a, x_{i}\right)$ and $Y_{i}=\left(c, a, y_{i}\right)$ (so that $\left(X_{i}\right)$ and $\left(Y_{i}\right)$ are rays in $(Q, \rho)$ ). Then, $\rho\left(X_{i}, Y_{i}\right) \preceq \rho\left(X_{0}, Y_{0}\right)$ for all $i \in \mathbb{N}$.

Proof. We can suppose that $i \gg 0$, so that $\left(a x_{i} \mid b x_{0}\right) \gg 0$ and $\left(a y_{i} \mid c y_{0}\right) \gg 0$. Let $(\tau, d)$ be an approximating tree for the set $\left\{a, b, c, x_{0}, y_{0}, x_{i}, y_{i}\right\}$. Let $x_{0}^{\prime}, x_{i}^{\prime}, y_{0}^{\prime}, y_{i}^{\prime} \in \tau$ be, respectively, the medians of $X_{0}, X_{i}, Y_{0}, Y_{i}$. Since $\left(a x_{i} \mid b x_{0}\right) \gg 0$, we have $\left(a x_{i} \mid b x_{0}\right) \geq 0$, and so $x_{i}^{\prime} \in\left[a, x_{0}^{\prime}\right]$. Similarly, $y_{i}^{\prime} \in\left[c, y_{0}^{\prime}\right]$. Also $d\left(x_{i}^{\prime}, x_{0}^{\prime}\right) \simeq$ $\rho\left(X_{i}, X_{0}\right) \simeq i \simeq d\left(y_{i}^{\prime}, y_{0}^{\prime}\right)$. It follows easily that $d\left(x_{i}^{\prime}, y_{i}^{\prime}\right) \preceq d\left(x_{0}^{\prime}, y_{0}^{\prime}\right)$, and so $\rho\left(X_{i}, Y_{i}\right) \preceq \rho\left(X_{0}, Y_{0}\right)$ as required.

In particular, we see that the rays $\left(X_{i}\right)$ and $\left(Y_{i}\right)$ are parallel. Thus, given any $a \in M$, we can define $f(a) \in \partial Q$ to be the ideal endpoint of a ray of this type. This gives us a map $f: M \longrightarrow \partial Q$. Note that if $a, b \in M$ are distinct, then Lemma 2.8 gives us a bi-infinite geodesic, $\left(X_{i}\right)_{i \in \mathbb{Z}}$, such that $\left(X_{i}\right)_{i \in \mathbb{N}}$ and $\left(X_{-i}\right)_{i \in \mathbb{N}}$ are each rays of this type, corresponding respectively to $a$ and $b$. It follows that $f(a) \neq f(b)$. This shows that $f$ is injective.

To verify the assertion about crossratios, consider distinct points, $x, y, z, w \in M$. Choose sequences, $x_{i} \rightarrow x, y_{i} \rightarrow y, z_{i} \rightarrow z$ and $w_{i} \rightarrow w$, so that $\left(y,\left(x_{i}\right)\right),\left(x,\left(y_{i}\right)\right)$, $\left(w,\left(z_{i}\right)\right)$ and $\left(z,\left(w_{i}\right)\right)$ are boundary rays. Let $X_{i}=\left(y, x, x_{i}\right), Y_{i}=\left(x, y, y_{i}\right), Z_{i}=$ $\left(w, z, z_{i}\right)$ and $W_{i}=\left(z, w, w_{i}\right)$. Thus, $\left(X_{i}\right),\left(Y_{i}\right),\left(Z_{i}\right)$ and $\left(W_{i}\right)$ are geodesic rays, tending, by definition, to $f(x), f(y), f(z)$ and $f(w)$ respectively. Let $(\tau, d)$ be an approximating tree for $\left\{x, y, w, z, x_{i}, y_{i}, z_{i}, w_{i}\right\}$. For sufficiently large $i$, we have $\left(x x_{i} \mid y z\right) \gg 0,\left(x x_{i} \mid z w\right) \gg 0$ and $\left(x x_{i} \mid w y\right) \gg 0$. Thus, we can suppose that $\left[x, x_{i}\right]$ does not meet $[y, z] \cup[z, w] \cup[w, y]$. We can make similar assumptions about the segments, $\left[y, y_{i}\right],\left[z, z_{i}\right]$ and $\left[w, w_{i}\right]$, permuting $x, y, z$ and $w$. Let $x^{\prime}, y^{\prime}, z^{\prime}, w^{\prime} \in \tau$ be the medians, respectively, of $X_{i}, Y_{i}, Z_{i}$ and $W_{i}$. We see that $x^{\prime}$ is the unique internal vertex of $\tau$ lying in the segment $\left[x, x_{i}\right]$, and similarly for $y^{\prime}, z^{\prime}$ and $w^{\prime}$. It follows that $\left(x^{\prime} y^{\prime} \mid z^{\prime} w^{\prime}\right)_{\tau}=(x y \mid z w)_{\tau}$. Now, $\rho\left(X_{i}, Y_{i}\right) \simeq d\left(x^{\prime}, y^{\prime}\right)$, etc. and so, $\left(X_{i} Y_{i} \mid Z_{i} W_{i}\right)_{\rho} \simeq\left(x^{\prime} y^{\prime} \mid z^{\prime} w^{\prime}\right)_{\tau}=(x y \mid z w)_{\tau} \simeq(x y \mid z w)$. In other words, we have $\left(X_{i} Y_{i} \mid Z_{i} W_{i}\right)_{\rho}=(x y \mid z w)$ for all sufficiently large $i$. From the definition of $(. . \mid . .)_{\rho}$ on $\partial Q$ as described in Section 3, we see that $(f(x) f(y) \mid f(z) f(w))_{\rho} \simeq(x y \mid z w)$. 
It remains to verify that $f(M)$ is dense in $\partial Q$. We first make a few general observations.

Suppose $W=(x, y, z) \in Q$. By Lemma 2.8, there is a bi-infinite sequence, $\left(x_{i}\right)_{i \in \mathbb{Z}}$, with $x_{0}=z$, and such that $X_{i} \rightarrow f(x)$ and $X_{-i} \rightarrow f(y)$, where $X_{i}=$ $\left(x, y, x_{i}\right)$ (so that $\left.X_{0}=W\right)$. Moreover, $\rho\left(X_{i}, X_{-i}\right) \simeq 2 i \simeq \rho\left(W, X_{i}\right)+\rho\left(W, X_{-i}\right)$. In other words, $\left(X_{i} X_{-i} \mid W W\right)_{\rho} \simeq 0$. As discussed in Section 3, it follows that $(f(x) f(y) \mid W W)_{\rho} \simeq 0$. Similarly, we have

$$
(f(y) f(z) \mid W W)_{\rho} \simeq 0 \quad \text { and } \quad(f(z) f(x) \mid W W)_{\rho} \simeq 0 .
$$

It follows that $W$ is a centre of the triple $(f(x), f(y), f(z))$. We have shown:

Lemma 4.6. Given distinct $x, y, z \in M$, the triple $(x, y, z)$ is a centre, in $Q$, of the triple of ideal points $(f(x), f(y), f(z))$.

We can now show that $f(M)$ is dense in $\partial Q$. To see this, suppose $w \in \partial Q$. Choose any sequence of points, $\left(W_{i}\right)_{i \in \mathbb{N}}$, of $Q$ tending to $w$. By Lemma 4.6, each $W_{i}$ is the centre of some triple, say $\left(a_{i}, b_{i}, c_{i}\right)$, of points of $f(M)$. Now, a simple geometric argument shows that, without loss of generality, $a_{i} \rightarrow w$.

We shall now turn our attention to compact spaces. Suppose that $M$ is a metrisable compactum, and that (..|..) is a crossratio on $M$.

Definition. We say that the crossratio (..|..) is compatible with the topology on $M$ if, given any distinct $x, y, z \in M$ and any sequence $x_{i} \rightarrow x$, we have $\left(x x_{i} \mid y z\right) \rightarrow \infty$.

Since we are assuming that $M$ is metrisable, this is the same as saying that the crossratio topology is coarser than the given topology. Since the given topology is compact and the crossratio topology is hausdorff, it follows that the two topologies must, in fact, coincide.

Suppose, now, that $M$ is perfect, and that (..|..) is a hyperbolic path crossratio. It follows that $(. . \mid .$.$) is also perfect. Let (Q, \rho)$ be the set of distinct triples, with induced quasimetric, $\rho$, and with $M$ embedded as a dense subset of $\partial Q$. Since $M$ is compact, it follows that, in fact, $M=\partial Q$. In summary, we have shown:

Proposition 4.7. Suppose that $M$ is a perfect metrisable compactum with compatible hyperbolic path crossratio, (..|..). One can put a natural hyperbolic path quasimetric, $\rho$, on the space of distinct triples, $Q$, of $M$, in such a way that $M$ is naturally identified by a homeomorphism with the boundary, $\partial Q$, of $Q$. Moreover, the crossratios (..|..) and (..|..) $)_{\rho}$ on $\partial Q$ differ by at most an additive constant.

This is all we will need to know for the proof of the main theorem, though a few comments are in order. We note that, since $Q$ is not locally finite, there is no a-priori reason to expect $\partial Q$ to be compact - as turns out to be the case. In fact, $Q$ is necessarily almost locally finite (i.e. quasi-isometric to a locally finite quasimetric space, as discussed in Section 3). In retrospect, knowing that $\partial Q$ is compact, this follows from Lemma 4.6 and Proposition 3.5. Indeed, we see that $Q$ contains a locally finite quasidense subset. In summary, it follows:

Proposition 4.8. Suppose that $M$ is a perfect metrisable compactum with compatible hyperbolic path crossratio (.....). Then there is a locally finite hyperbolic path quasimetric space, $(P, \rho)$, and a homomorphism of $M$ onto $\partial P$, such that (..|..) agrees with $(. . \mid . .)_{\rho}$ on $\partial P$ up to at most an additive constant. 


\section{More about Crossratios}

In this section, we give an alternative formulation of a hyperbolic crossratio.

Suppose that (..|..) is a crossratio on a set $M$. Given five distinct points, $a, b, x, y, z \in M$, let $(a b \mid x y z)=\min \{(a b \mid x y),(a b \mid y z),(a b \mid z x)\}$. This is symmetric under any permutation of $\{a, b\}$ or $\{x, y, z\}$. We write $(x y z \mid a b)=(a b \mid x y z)$. If (.....) is hyperbolic, we see that $(x y \mid z w) \simeq(x y a \mid z w)+(x y \mid z w a)$ for any $(x, y, z, w, a) \in$ $\Theta_{5}(M)$. It turns out that this can be used to characterise hyperbolicity of crossratios.

Suppose then that $M$ is a set, and (..|..) is a crossratio on $M$ (i.e. a map $[(x, y, z, w) \mapsto(x y \mid z w)]: \Theta_{4}(M) \longrightarrow[0, \infty)$ satisfying $(x y \mid z w)=(y x \mid z w)=$ $(z w \mid x y)$ for all $\left.(x, y, z, w) \in \Theta_{4}(M)\right)$. Suppose we also have a map $[(a, b, x, y, z) \mapsto$ $(a b \mid x y z)]: \Theta_{5}(M) \longrightarrow[0, \infty)$ satisfying $(a b \mid x y z)=(b a \mid x y z)=(a b \mid y x z)=(a b \mid x z y)$ for all $(a, b, x, y, z) \in \Theta_{5}(M)$. We write $(x y z \mid a b)=(a b \mid x y z)$. Suppose that there is some $k \geq 0$ such that:

(C1): If $(x, y, z, w) \in \Theta_{4}(M)$, then at least two of the three quantities $(x y \mid z w)$, $(x z \mid y w)$ and $(x w \mid y z)$ are at most $k$, and

$\left(\mathrm{C} 2^{\prime}\right)$ : If $(x, y, z, w, a) \in \Theta_{5}(M)$, then

$$
(x y \mid z w) \simeq_{k}(x y a \mid z w)+(x y \mid z w a) .
$$

Note that $\left(\mathrm{C} 2^{\prime}\right)$ implies that $(x y a \mid z w) \preceq(x y \mid z w)$.

Proposition 5.1. A crossratio arising in this way is hyperbolic.

Proof. We need to verify property (C2). Suppose, then, that $x, y, z, w, a \in M$ are distinct. We can suppose that $(x y \mid z w)$ is maximal among all values of the crossratio restricted to the set $\{x, y, z, w, a\}$. We claim that $(x y: a: z w)$ holds.

Let $p=(x y \mid z w a)$ and $q=(x y a \mid z w)$. Thus, $(x y \mid z w) \simeq p+q$. If $p, q \simeq 0$, then $(x y \mid z w) \simeq 0$ and so all crossratios on this five point set are $\simeq 0$, so there is nothing to prove. We can thus suppose that $p \gg 0$.

Now, $p=(x y \mid z w a) \preceq(x y \mid a z)$, and so $(x y \mid a z) \gg 0$. By $(\mathrm{C} 1)$, we see that $(a x \mid y z) \simeq 0$ and so $(a x \mid z w y) \simeq 0$. It follows that $(a x \mid z w) \simeq(a x y \mid z w)+(a x \mid z w y) \simeq$ $(a x y \mid z w)=q$. Interchanging $x$ and $y$, we get $(a y \mid z w) \simeq(a x y \mid z w)=q$. Again, since $(x y \mid a z) \gg 0$, we get, by $(\mathrm{C} 1)$, that $(x a \mid y z) \simeq 0$ and $(y a \mid x z) \simeq 0$. Similarly, interchanging $z$ and $w$, we get $(x a \mid y w) \simeq 0$ and $(y a \mid x w) \simeq 0$. We also note that since $(x y \mid z w) \succeq p \gg 0$, we get $(x z \mid y w) \simeq 0$ and $(x w \mid y z) \simeq 0$.

For the remaining inequalities, we consider two cases, namely $q \gg 0$ and $q \simeq 0$.

If $q \gg 0$, then all the remaining inequalities follow by symmetry, interchanging $\{x, y\}$ with $\{z, w\}$ and $p$ with $q$.

We can thus suppose that $q \simeq 0$. Thus, $(x y \mid z w) \simeq p+0=(x y \mid z w a)$. Now, by maximality of $(x y \mid z w)$, we have $(x y \mid z w) \geq(x y \mid a w) \simeq(x y z \mid a w)+(x y \mid a w z) \simeq$ $(x y z \mid a w)+(x y \mid z w)$, and so $(x y z \mid a w) \simeq 0$. It follows that $(x y \mid a w) \simeq(x y z \mid a w)+$ $(x y \mid a w z) \simeq 0+p=p$. Similarly, interchanging $z$ and $w$, we see that $(x y \mid a z) \simeq p$.

We already know that $(x a \mid y w) \simeq 0$, and so $(x a \mid z w y) \simeq 0$. Thus, $(x a \mid z w) \simeq$ $(x a y \mid z w)+(x a \mid z w y) \simeq 0$. Similarly, interchanging $x$ and $y$, we get $(y a \mid z w) \simeq 0$.

We also know that $(y a \mid x z) \simeq 0$, and so $($ way $\mid x z) \simeq 0$. We have already shown that $(x y z \mid a w) \simeq 0$. Thus, $(w a \mid x z) \simeq(w a y \mid x z)+(w a \mid x z y) \simeq 0$. Similarly (interchanging $x$ with $y$ and $z$ with $w)$ we get $(w a \mid y z) \simeq 0,(z a \mid x w) \simeq 0$ and $(w a \mid y z) \simeq 0$.

This deals with all values of the crossratio (up to its symmetry) and so we deduce that $(x y: a: z w)$ as claimed. 
As a consequence of the argument, we note:

Lemma 5.2. Suppose that $M$ and (..|..) satisfy the hypotheses of Proposition 5.1, together with the hypothesis that given any $(x, y, z, w) \in \Theta_{4}(M)$ and any $p \leq$ $(x y \mid z w)$ we can find some $a \in M$ such that $(x y \mid z w a) \simeq p$. Then (..|..) is a hyperbolic path crossratio.

\section{Systems of AnNuli}

We can think of a hyperbolic crossratio on a compactum, $M$, as defining a kind of "quasiconformal structure". Another way to formulate this idea might to specify a collection of "annuli" in $M$, which we might imagine as having moduli bounded away from 0 and $\infty$. The principal condition we should require of such a collection is one which rules out too many transverse crossings of one set of nested annuli by another (see axiom (A2) below). The main aim of this section will be to describe how such a system gives rise to a hyperbolic crossratio. There are no doubt other directions one could explore concerning the relationships between these notions, but they will not be directly relevant to the main results of this paper.

(We remark that notions of quasiconformal structure for connected metric measure spaces have been studied in [HK]. To what extent these ideas might be applicable, for example, to one-ended hyperbolic groups, remains to be explored. A more combinatorial approach to quasiconformal structures, via automatic structures, is central to the progress Cannon and his coworkers have made in understanding convergence actions on the 2 -sphere - see [CanS].)

The notion of an "annulus" is central to the construction of the JSJ decomposition in [Bo3]. We shall quote some of the elementary results of that paper. Since we are not concerned here with the connectedness properties of our compactum, we shall omit some of the clauses from the definition of an "annulus" as given in that paper.

Let $M$ be a compactum.

Definition. An annulus, $A$, is an ordered pair, $\left(A^{-}, A^{+}\right)$, of disjoint closed subsets of $M$ such that $M \backslash\left(A^{-} \cup A^{+}\right) \neq \emptyset$.

By an annulus system, $\mathcal{A}$, we simply mean a set of such annuli. Given an annulus, $A=\left(A^{-}, A^{+}\right)$, we shall write $-A=\left(A^{+}, A^{-}\right)$. We shall say that an annulus system, $\mathcal{A}$, is symmetric if $A \in \mathcal{A}$.

Given a closed set, $K \subseteq M$, and an annulus, $A$, we shall write $K<A$ to mean that $K \subseteq \operatorname{int} A^{-}$. We write $A<K$ to mean that $K<-A$, or equivalently, that $K \subseteq \operatorname{int} A^{+}$. Given annuli $A, B$ we write $A<B$ to mean that $M=\operatorname{int} A^{-} \cup \operatorname{int} B^{+}$ (or equivalently, $M \backslash \operatorname{int} A^{+}<B$, or $M \backslash \operatorname{int} B^{-}>A$ ). Clearly, $A<B$ if and only if $-B<-A$. We note that $<$ defines a partial order on the set of annuli.

We now fix an annulus system, $\mathcal{A}$. Given closed subsets, $K, L \subseteq M$, we write $(K \mid L) \in \mathbb{N} \cup\{\infty\}$ for the maximal number, $n \in \mathbb{N}$, such that we can find annuli $A_{1}, A_{2}, \ldots, A_{n} \in \mathcal{A}$ such that

$$
K<A_{1}<A_{2}<\cdots<A_{n}<L .
$$

We set $(K \mid L)=\infty$ if there is no such bound.

We refer to $\left(A_{i}\right)_{i}$ as a sequence of nested annuli separating $K$ and $L$. (For the purposes of this paper, one could probably take the alternative approach of simply defining $(K \mid L)$ to be the total number of annuli of $\mathcal{A}$ which individually separate 
$K$ from $L$. However, the route we have chosen seems more intuitive, technically simpler, and ties in better with [Bo3].)

By the symmetry of $\mathcal{A}$, we see that $(K \mid L)=(L \mid K)$. Also, if $K^{\prime} \subseteq K$, then $(K \mid L) \leq\left(K^{\prime} \mid L\right)$. Clearly, if $(K \mid L)>0$, then $K \cap L=\emptyset$. We also note:

Lemma 6.1. Given any closed subsets, $K, L \subseteq M$, and any $a \in M$, we have

$$
(K \mid L) \leq(K \cup\{a\} \mid L)+(K \mid L \cup\{a\})+1 .
$$

Proof. Suppose that $(K \mid L)=n \in \mathbb{N}$. There are annuli $A_{1}, \ldots, A_{n} \in \mathcal{A}$ with $K<A_{1}<\cdots<A_{n}<L$. Let $m=\max \left\{i \mid A_{i}<a\right\}$. Thus, $K<A_{1}<\cdots<A_{m}<$ $L \cup\{a\}$, so $(K \mid L \cup\{a\}) \geq m$. If $m \geq n-1$, we are done. Otherwise, $a \notin \operatorname{int} A_{m+1}^{+}$ and so $a \in \operatorname{int} A_{m+2}^{-}$since $A_{m+1}<A_{m+2}$. Thus $K \cup\{a\}<A_{m+2}<\cdots<A_{n}<L$, so $(K \cup\{a\} \mid L) \geq n-m-1$. The result follows in the case where $(K \mid L)$ is finite.

If $(K \mid L)=\infty$, the same argument shows that either $(K \cup\{a\} \mid L)=\infty$ or $(K \mid L \cup\{a\})=\infty$.

If $K=\left\{x_{1}, x_{2}, \ldots, x_{n}\right\}$, then we shall abbreviate $\left(\left\{x_{1}, x_{2}, \ldots, x_{n}\right\} \mid L\right)$ as $\left(x_{1} x_{2} \ldots x_{n} \mid L\right)$, and similarly for $L$. In particular, $(x y \mid z w)=(\{x, y\} \mid\{z, w\})$. Note that the map $[(x, y, z, w) \mapsto(x y \mid z w)]$, restricted to $\Theta_{4}(M)$, is a crossratio on $M$. We shall assume that

(A1): If $x \neq y$ and $z \neq w$, then $(x y \mid z w)<\infty$.

(A2): There is some $k \geq 0$ such that there are no four points, $x, y, z, w \in M$, with $(x z \mid y w)>k$ and $(x w \mid y z)>k$.

Thus, axiom (A2) is just a rephrasing of axiom (C1) for hyperbolic crossratios.

(As an example, consider the case where $M$ is the Riemann sphere, $S^{2}$, and $\mathcal{A}$ is an annulus system such that $A^{+}$and $A^{-}$are both connected for all $A \in \mathcal{A}$. We intuitively think of the annulus, $A$, as the annular region $S^{2} \backslash\left(A^{+} \cup A^{-}\right)$. If the analytic moduli of these annular regions are bounded away from 0 and $\infty$ as $A$ ranges over $\mathcal{A}$, then the system $\mathcal{A}$ will necessarily satisfy axioms (A1) and (A2).)

Lemma 6.2. Suppose that $\mathcal{A}$ is a symmetric annulus system satisfying axioms (A1) and (A2). If $K, L \subseteq M$ are closed and nonempty, and $a \in M$, then

$$
(K \cup\{a\} \mid L)+(K \mid L \cup\{a\}) \leq(K \mid L)+2 k+2 .
$$

Proof. Let $(K \mid L \cup\{a\})=p,(K \cup\{a\} \mid L)=q$ and $(K \mid L)=n$. Thus, $p \leq n$ and $q \leq n$. Suppose, for contradiction, that $p+q>n+2 k+2$. It follows that $p, q \geq k+2$. Let $r=p-k-1$ and $s=q-k-1$. Thus, $r, s \geq 1$.

We can find annuli $A_{1}, \ldots, A_{p}, B_{1}, \ldots, B_{q} \in \mathcal{A}$ such that

$$
K<A_{1}<\cdots<A_{p}<L \cup\{a\}
$$

and

$$
L<B_{1}<\cdots<B_{q}<K \cup\{a\} .
$$

We claim that $A_{r}<-B_{s}$.

Suppose not. Then $M \neq \operatorname{int} A_{r}^{+} \cup \operatorname{int} B_{s}^{+}$, so there exists $b \in M \backslash\left(\operatorname{int} A_{r}^{+} \cup \operatorname{int} B_{s}^{+}\right)$. Since $A_{r}<A_{r+1}$ and $B_{s}<B_{s+1}$, we get $b \in \operatorname{int} A_{r+1}^{-} \cap \operatorname{int} B_{s+1}^{-}$, in other words, $b<A_{r+1}$ and $b<B_{s+1}$. It follows that

$$
K \cup\{b\}<A_{r+1}<\cdots<A_{p}<L \cup\{a\}
$$

and

$$
L \cup\{b\}<B_{s+1}<\cdots<B_{q}<K \cup\{a\},
$$


and so $(K \cup\{b\} \mid L \cup\{a\}) \geq k+1$ and $(L \cup\{b\} \mid K \cup\{a\}) \geq k+1$. Now, choosing any $x \in K$ and $y \in L$, we get $(x b \mid y a)>k$ and $(y b \mid x a)>k$, contradicting axiom (A2). This proves the claim that $A_{r}<-B_{s}$.

But now, we have

$$
K<A_{1}<A_{2}<\cdots<A_{r}<-B_{s}<\cdots<-B_{2}<-B_{1}<L,
$$

and so $n=(K \mid L) \geq r+s=p+q-2 k-2$, proving the result.

Putting Lemmas 6.1 and 6.2 together, we get:

Lemma 6.3. If $K, L \subseteq M$ are closed and nonempty, and $a \in M$, then

$$
(K \mid L) \simeq(K \cup\{a\} \mid L)+(K \mid L \cup\{a\}) .
$$

In particular, we see that if $(x, y, z, w, a) \in \Theta_{5}(M)$, then $(x y \mid z w) \simeq(x y a \mid z w)+$ $(x y \mid z w a)$. But this is precisely axiom $\left(\mathrm{C}^{\prime}\right)$ described in Section 5 . Thus, by Proposition 5.1, we deduce that (..|..) is a hyperbolic crossratio.

Moreover, we have

Lemma 6.4. Given $K, L \subseteq M$ closed and nonempty, and $p \leq(K \mid L)$, there is some $a \in M$ with $(K \mid L \cup\{a\}) \simeq p$.

Proof. Let $n=(K \mid L)$ and choose $A_{1}, \ldots, A_{n} \in \mathcal{A}$ so that $K<A_{1}<\cdots<A_{n}<L$. By the definition of an annulus, we can find some $a \in M \backslash\left(\operatorname{int} A_{p}^{-} \cup \operatorname{int} A_{p}^{+}\right.$). Since $A_{p-1}<A_{p}$, we see that $a \in \operatorname{int} A_{p-1}^{+}$, in other words, $a>A_{p-1}$. Thus, $K<A_{1}<\cdots<A_{p-1}<L \cup\{a\}$, and so $(K \mid L \cup\{a\}) \geq p-1$. Similarly, we get $(K \cup\{a\} \mid L) \geq n-p$. But, by Lemma 6.3, we have $n \simeq(K \cup\{a\} \mid L)+(K \mid L \cup\{a\})$, and so $(K \mid L \cup\{a\}) \simeq p$ as required.

In particular, we see that the hypotheses of Lemma 5.2 are satisfied, and so (..|..) is a path crossratio.

In summary, we have shown:

Proposition 6.5. Suppose that $M$ is a compactum, and $\mathcal{A}$ is a symmetric annulus system on $M$ satisfying axioms (A1) and (A2). Then, the map $[(x, y, z, w) \mapsto$ $(x y \mid z w)]: \Theta_{4}(M) \longrightarrow[0, \infty)$ defined as above (as the maximum number of nested annuli of $\mathcal{A}$ separating $\{x, y\}$ from $\{z, w\})$ is a hyperbolic path crossratio on $M$.

This gives us a hyperbolic path quasimetric on the set of distinct triples as described in Section 4. To go further, we shall want to assume that $M$ is perfect, and that:

(A3): If $x, y, z \in M$ are distinct, then $(x \mid y z)=\infty$.

Lemma 6.6. If $\mathcal{A}$ satisfies (A3), then the crossratio (..|..) is compatible with the topology on $M$.

Proof. Suppose that $x, y, z \in M$ are distinct, and $x_{i} \in M \backslash\{x, y, z\}$ is a sequence converging on $x$. We want to show that $\left(x x_{i} \mid y z\right) \rightarrow \infty$.

Given any $n \in \mathbb{N}$, we can find $A_{1}, \ldots, A_{n} \in \mathcal{A}$ with $\{y, z\}<A_{1}<\cdots<A_{n}<$ $\{x\}$. Thus, $x \in \operatorname{int} A_{n}^{+}$. So, for sufficiently large $i$, we have $x_{i} \in \operatorname{int} A_{n}^{+}$and so $\left(x x_{i} \mid y z\right) \geq n$.

Thus, if $\mathcal{A}$ satisfies axioms (A1), (A2) and (A3), we obtain all the results of Section 4. In particular, we can put a natural hyperbolic path quasimetric, $\rho$, on 
the the set, $Q$, of distinct triples, so that $M$ is identified by homeomorphism with $\partial Q$.

We note that axiom (A3) is implied by the following axiom:

$\left(\mathrm{A} 3^{\prime}\right)$ : If $K \subseteq M$ is closed and $x \in M \backslash K$, then $(K \mid x)>0$.

In other words, there is an annulus, $A_{1} \in \mathcal{A}$, with $K<A_{1}<x$. Now, $x \in \operatorname{int} A_{1}^{+}$, so we can apply $\left(\mathrm{A} 3^{\prime}\right)$ again, with $M \backslash \operatorname{int} A_{1}^{+}$replacing $K$, to give another annulus, $A_{2} \in \mathcal{A}$, with $M \backslash \operatorname{int} A_{1}^{+}<A_{2}<x$. In other words, $K<A_{1}<A_{2}<x$. Continuing inductively in this fashion, we obtain an infinite sequence of nested annuli separating $K$ from $x$, and so, certainly, $(K \mid x)=\infty$. This shows that axiom (A3') implies axiom (A3).

\section{Convergence groups and CROssratios}

In this section, we show how a convergence action on a compactum, $M$, gives rise to an annulus system, and hence to a crossratio. We conclude with a proof of Theorem 0.1. In this context, the set of distinct triples of $M$ will appear in two slightly different roles: one as the topological space featuring in the hypothesis (for which we shall retain the notation $\Theta_{3}(M)$ ) and one as the set (which we denote by $Q)$ on which we shall construct a hyperbolic quasimetric.

Let $M$ be a metrisable compactum, and let $\Gamma$ be a group action by homeomorphism on $M$. Let $\Theta_{3}(M)$ be the space of distinct triples of $M$ (with topology induced from the product topology on $M^{3}$ ). Thus, $\Theta_{3}(M)$ is locally compact and metrisable. We get an induced action of $\Gamma$ on $\Theta_{3}(M)$. We say that $\Gamma$ is a convergence group if the action on $\Theta_{3}(M)$ is properly discontinuous (i.e. for any compact subset, $K \subseteq \Theta_{3}(M)$, the set $\{\gamma \in \Gamma \mid \gamma K \cap K \neq \emptyset\}$ is finite).

Proposition 7.1. $\Gamma$ is a convergence group if and only if the following condition holds. If $\left(\gamma_{i}\right)_{i \in \mathbb{N}}$ is an infinite sequence of distinct elements of $\Gamma$, then we can find points $a, b \in M$, and a subsequence, $\left(\gamma_{j}\right)_{j}$, of $\left(\gamma_{i}\right)_{i}$, such that the maps $\gamma_{j} \mid M \backslash\{a\}$ converge locally uniformly to $b$.

In fact, the condition given by Proposition 7.1 is the original formulation of the notion of convergence group as described in [GeM1]. A proof of this equivalence can be found in [Bo4] (generalising the argument of [GeM2] in the case where $M$ is a sphere). For further discussion of convergence groups in connection with hyperbolic groups, see also [T2] and [F].

Now, suppose that $\Gamma$ acts as a convergence group on $M$, and that $\mathcal{A}$ is a $\Gamma$ invariant symmetric annulus system, with $\mathcal{A} / \Gamma$ finite (i.e. $\mathcal{A}$ is the union of the $\Gamma$-orbits of a finite set of annuli).

Lemma 7.2. Suppose that $x, y, z, w \in M$. Suppose that $\left(x_{i}\right)_{i},\left(y_{i}\right)_{i},\left(z_{i}\right)_{i}$ and $\left(w_{i}\right)_{i}$ are sequences in $M$ converging, respectively, to $x, y, z$ and $w$. Suppose that we have a sequence of annuli, $\left(A_{i}\right)_{i}$, such that $\left\{x_{i}, y_{i}\right\}<A_{i}<\left\{z_{i}, w_{i}\right\}$ for all $i$, and such that the set $\left\{A_{i} \mid i \in \mathbb{N}\right\}$ is infinite. Then, either $x=y$ or $z=w$.

Proof. Since $\mathcal{A} / \Gamma$ is finite, we can suppose, after passing to a subsequence, that there is a fixed annulus, $A \in \mathcal{A}$, and a sequence of distinct elements, $\left(\gamma_{i}\right)_{i}$, of $\Gamma$, such that $A_{i}=\gamma_{i} A$. Passing to a further subsequence, we can find points $a, b \in M$ such that $\gamma_{i} \mid M \backslash\{a\}$ converges locally uniformly to $b$. Without loss of generality (interchanging $\{x, y\}$ with $\{z, w\}$ and $A$ with $-A$ if necessary) we can suppose that $a \notin A^{+}$. Thus, $\gamma_{i} \mid A^{+}$converges uniformly to $b$. In other words, given any open neighbourhood, $U$, of $b$, we have $A_{i}^{+}=\gamma_{i} A^{+} \subseteq U$ for all sufficiently large $i$. But 
$A_{i}<\left\{z_{i}, w_{i}\right\}$, so $z_{i}, w_{i} \in A_{i}^{+} \subseteq U$. Thus, $z_{i} \rightarrow b$ and $w_{i} \rightarrow b$, showing that $z=w=b$.

Recall the notation $(x y \mid z w)$, introduced in Section 6 , for the maximal length of a chain of nested annuli separating $\{x, y\}$ from $\{z, w\}$. This is clearly $\Gamma$-invariant.

Lemma 7.3. Suppose that $x, y, z, w \in M$ and that there exist sequences, $x_{i} \rightarrow x$, $y_{i} \rightarrow y, z_{i} \rightarrow z$ and $w_{i} \rightarrow w$, with $\left(x_{i} y_{i} \mid z_{i} w_{i}\right) \rightarrow \infty$. Then either $x=z$ or $y=w$.

Proof. The hypotheses of Lemma 7.2 are clearly satisfied in this case.

We immediately deduce that $(x y \mid z w)<\infty$ for all $(x, y, z, w) \in \Theta_{4}(M)$. In other words, axiom (A1) of Section 6 is satisfied.

Lemma 7.4. There is some $k \geq 0$ such that if $(x, y, z, w) \in \Theta_{4}(M)$ with $(x y \mid z w) \geq$ $k$, then $(x z \mid y w)=0$.

Proof. Suppose not. We can find a sequence, $\left(\left(x_{i}, y_{i}, z_{i}, w_{i}\right)\right)_{i}$, of points of $\Theta_{4}(M)$ with $\left(x_{i} y_{i} \mid z_{i} w_{i}\right) \rightarrow \infty$ and with $\left(x_{i} z_{i} \mid y_{i} w_{i}\right)>0$ for all $i$. Thus, for all $i$, there is an annulus, $A_{i} \in \mathcal{A}$, with $\left\{x_{i}, z_{i}\right\}<A_{i}<\left\{y_{i}, w_{i}\right\}$. Since $\mathcal{A} / \Gamma$ is finite, we can suppose (after passing to a subsequence) that each $A_{i}$ is the image under $\Gamma$ of some fixed $A \in \mathcal{A}$. Now since (..|..) is $\Gamma$-invariant, we can suppose (after translating under $\Gamma)$ that $\left\{x_{i}, z_{i}\right\}<A<\left\{y_{i}, w_{i}\right\}$ for all $i$. Again after passing to a subsequence, we can assume that the sequences $\left(x_{i}\right)_{i},\left(y_{i}\right)_{i},\left(z_{i}\right)_{i}$ and $\left(w_{i}\right)_{i}$ converge, respectively, to points $x, y, z, w \in M$. Now, $x, z \in A^{-}$and $y, w \in A^{+}$, and so, in particular, $x \neq y$ and $z \neq w$. But since $\left(x_{i} y_{i} \mid z_{i} w_{i}\right) \rightarrow \infty$, this contradicts Lemma 7.3.

In particular, we see that axiom (A2) of Section 6 is satisfied. Thus, by Propositon $6.5,(. . \mid$.$) is a hyperbolic path crossratio. In summary, we have shown:$

Proposition 7.5. Suppose a group, $\Gamma$, acts as a convergence group on a metrisable compactum, $M$. If $\mathcal{A}$ is a $\Gamma$-invariant symmetric annulus system with $\mathcal{A} / \Gamma$ finite, then (.....) is a hyperbolic path crossratio on $M$.

To go further, we shall need to assume that $M$ is perfect, and that the action of $\Gamma$ on $\Theta_{3}(M)$ is also cocompact (i.e. $\Theta_{3}(M) / \Gamma$ is compact). This is easily seen to be equivalent to asserting that there is a compact subset, $\Theta_{0} \subseteq \Theta_{3}(M)$, with $\Theta_{3}(M)=\bigcup \Gamma \Theta_{0}$. In this case, we shall construct an annulus system, $\mathcal{A}$, for $M$, as follows. Here, we shall follow along the lines of [Bo3]. An alternative approach would be via Proposition 8.2. However, the latter is unnecessarily complicated for the present purposes (and also, as we have phrased it, makes essential use of metrisability).

Given $\theta=(x, y, z) \in \Theta_{3}(M)$, choose open subsets, $U(\theta), V(\theta)$ and $W(\theta)$, of $M$, containing $x, y$, and $z$ respectively, whose closures, $\bar{U}(\theta), \bar{V}(\theta)$ and $\bar{W}(\theta)$, are pairwise disjoint. Let $\Theta(\theta)=U(\theta) \times V(\theta) \times W(\theta) \subseteq \Theta_{3}(M)$. We now find a finite set $\theta_{1}, \ldots, \theta_{n} \in \Theta_{0}$, such that $\Theta_{0} \subseteq \bigcup_{i=1}^{m} \Theta\left(\theta_{i}\right)$. Let $A_{i}$ be the annulus $\left(\bar{U}\left(\theta_{i}\right), \bar{V}\left(\theta_{i}\right)\right)$. Let $\mathcal{A}$ be the set of annuli of the form $\gamma A_{i}$ or $-\gamma A_{i}$ as $\gamma$ ranges over $\Gamma$ and $i$ runs from 1 to $m$. Thus, $\mathcal{A}$ is symmetric and $\Gamma$-invariant, and $\mathcal{A} / \Gamma$ is finite.

Lemma 7.6. If $K \subseteq M$ is closed, and $x \in M \backslash K$, then there is some $A \in \mathcal{A}$ with $K<A<x$.

Proof. This is proven in [Bo3], though for completeness, we outline the argument below. The idea is to choose any $y \in M \backslash\{x\}$, and any sequence $\left(x_{i}\right)_{i}$ of points 
of $M \backslash\{x, y\}$ converging on $x$. We can thus find a sequence $\left(\gamma_{i}\right)_{i}$ in $\Gamma$, such that the triples $\gamma_{i}\left(x, y, x_{i}\right)$ lie in a compact subset of $\Theta_{3}(M)$, and so (after passing to a subsequence) converge on some $(a, b, c) \in \Theta_{3}(M)$. From the construction of $\mathcal{A}$, we can find some $B \in \mathcal{A}$ with $b<B<a$. Again after passing to a subsequence, we find that $\gamma_{i} \mid M \backslash\{x\}$ converges locally uniformly to $b$. Thus, for sufficiently large $i$, we have $\gamma_{i} K<B<\gamma_{i} x$, so $K<A<x$, where $A=\gamma_{i}^{-1} B \in \mathcal{A}$.

The conclusion of Lemma 7.6 is precisely axiom $\left(\mathrm{A} 3^{\prime}\right)$ of Section 6 , and so axiom (A3) follows.

By Lemma 6.6, the topology on $M$ is compatible with $(. . \mid .$.$) , and so agrees with$ the crossratio topology. Let $\rho$ be the quasimetric defined on the set $Q=\Theta_{3}(M)$, as constructed in Section 4. This is clearly $\Gamma$-invariant. By Proposition 4.7, $(Q, \rho)$ is a hyperbolic path quasimetric space, with $\partial Q$ naturally identified with $M$. By naturality, the action of $\Gamma$ commutes with this identification.

We still have to verify that $\Gamma$ is a hyperbolic group. To this end, we choose any $\theta \in Q$, and define a $\Gamma$-equivariant map, $f: \Gamma \longrightarrow Q$, by setting $f(\gamma)=\gamma \theta$. We pull back $\rho$ to give a left invariant quasimetric on $\Gamma$, which we shall also denote by $\rho$.

Lemma 7.7. $(\Gamma, \rho)$ is locally finite.

Proof. In other words, every bounded set is finite. To see this, recall that $\theta$ is the centre of three distinct points, $x, y, z \in M$ (see Lemma 4.6). Suppose that $\left(\gamma_{i}\right)_{i}$ is a sequence of distinct elements of $\Gamma$. After passing to a subsequence, we can find $a, b \in M$ with $\gamma_{i} \mid M \backslash\{a\}$ converging locally uniformly to $b$. We can suppose that $x, y \neq a$, and so $\gamma_{i} x \rightarrow b$ and $\gamma_{i} y \rightarrow b$. From this, it follows easily that $\rho\left(\theta, \gamma_{i} \theta\right) \rightarrow \infty$. Thus, $\left\{\gamma_{i} \mid i \in \mathbb{N}\right\}$ is unbounded in $(\Gamma, \rho)$.

Lemma 7.8. Every point of $Q$ lies a bounded distance from some point in the $\Gamma$-orbit of $\theta$.

Proof. If not, we could find a sequence, $\theta_{i} \in Q$, with $\rho\left(\theta_{i}, \Gamma \theta\right) \rightarrow \infty$. We know (Lemma 4.6) that $\theta_{i}$ is the centre of some triple $\left(x_{i}, y_{i}, z_{i}\right)$, of points of $M$. From the cocompactness assumption, we can assume that the triples $\left(x_{i}, y_{i}, z_{i}\right)$ lie in a compact subset of $\Theta_{3}(M)$. It now follows easily that the set $\left\{\theta_{i} \mid i \in \mathbb{N}\right\}$ is a bounded subset of $Q$, contradicting the fact that $\rho\left(\theta_{i}, \Gamma \theta\right) \rightarrow \infty$.

It therefore follows that the map $f$ is a quasi-isometry of $(\Gamma, \rho)$ to $(Q, \rho)$. Thus, $(\Gamma, \rho)$ is a hyperbolic path quasimetric space, and its boundary is naturally, and hence $\Gamma$-equivariantly, identified with $\partial Q$ and hence with $M$. Now, $(\Gamma, \rho)$ is hyperbolic, and so, by Lemma 3.3, $\Gamma$ is a hyperbolic group, and its Gromov boundary, $\partial \Gamma$, is naturally identified with the boundary of $(\Gamma, \rho)$. We thus get a $\Gamma$-equivariant homeomorphism of $\partial \Gamma$ to $M$. This proves Theorem 0.1 .

The converse of Theorem 0.1, namely that the action of a hyperbolic group, $\Gamma$, on $\Theta_{3}(\partial \Gamma)$ is properly discontinuous and cocompact, is well known and essentially elementary. A proof can be found, for example, in [Bo4]. Direct proofs that the action of $\Gamma$ on $\partial \Gamma$ satisfies the convergence hypothesis as formulated in [GeM1] (i.e. the conclusion of Proposition 7.1) can be found in [F] or [T2].

\section{A CHARACTERISATION USING CONICAL LIMIT POINTS}

In this section, we give an alternative characterisation of hyperbolic groups, which might be thought of as a stronger result. In doing so, we shall give a general 
construction of annulus systems for convergence groups, which might have applications elsewhere.

Let $M$ be a perfect metrisable compactum and $\Gamma$ be a convergence group acting on $M$. We show that if every point of $M$ is a conical limit point, then $M$ is a uniform convergence group. Another proof has been given by Tukia [T3]. Tukia gives a selfcontained argument based directly on the convergence group hypothesis. Here we give another argument based on the constructions of this paper. I'm indebted to Pekka Tukia, for sending me outlines of his proof, which inspired me to consider if such ideas could be phrased in more geometrical terms.

In this section, the metrisability of $M$ is used in a much more explicit way than in the rest of this paper. It is unclear whether or not this assumption can be dropped in this case. (Of course, one would expect to have to modify the definition of a conical limit point to use nets rather than sequences.)

Suppose then, that $\Gamma$ is a convergence group acting on $M$. There are a number of equivalent ways of defining a "conical limit point". Among them:

Definition. A point $x \in M$ is a conical limit point if there are distinct points, $b, c \in M$, and a sequence, $\left(\gamma_{i}\right)_{i}$ in $\Gamma$, such that $\gamma_{i} x \rightarrow b$ and $\gamma_{i} y \rightarrow c$ for all $y \in M \backslash\{x\}$.

(Of course, the convergence of the maps $\gamma_{i} \mid M \backslash\{x\}$ to the point $c$ can be assumed to be locally uniform.) Equivalently, we see that $x$ is a conical limit point if and only if there is some $a \in M \backslash\{x\}$, a sequence $\left(x_{i}\right)_{i}$ in $M \backslash\{x\}$, and a compact set $\Theta_{0} \subseteq \Theta_{3}(M)$ such that $\left(a, x, x_{i}\right) \in \bigcup \Gamma \Theta_{0}$ for all $i \in \mathbb{N}$. For further discussion of conical limit points in this context, see for example, [Bo4] [T3].

Conical limit points play an important role in the theory of Kleinian groups. (They are also known as "radial limit points" or as "points of approximation".) They were originally introduced by Heglund, and they have been used by many authors since. In the particular context of convergence groups they have been studied by Martin and Tukia; see for example, [MT].

The main result of this section is:

Theorem 8.1. Suppose that $M$ is a perfect metrisable compactum, and that $\Gamma$ is a group with a convergence action on $M$. If every point of $M$ is a conical limit point, then $\Gamma$ is a uniform convergence group (and hence hyperbolic).

(Note that it follows, in retrospect, that the set $\Theta_{0}$ featuring in the earlier description of a conical limit point, $x$, can in fact be chosen independently of the point $x \in M$. This is equivalent to the assumption that convergence to conical limit points is "uniform" in the sense described in $[\mathrm{BeM}]$. If we were to take this as an additional hypothesis, it will be seen that the proof given below can be simplified.)

We begin in a fairly general context. Let $M$ be a perfect metrisable compactum. We fix some metric, $d$, on $M$ (inducing the given topology). Given a subset, $K \subseteq M$, write $\operatorname{diam}(K)$ for its diameter in this metric. If $A=\left(A^{-}, A^{+}\right)$is an annulus, we write $\lambda(A)=\min \left\{\operatorname{diam}\left(A^{-}\right), \operatorname{diam}\left(A^{+}\right)\right\}$and $\mu(A)=d\left(A^{-}, A^{+}\right)$. Suppose that $\Gamma$ is a convergence group, and that $\mathcal{B}$ is a $\Gamma$-invariant annulus system with $\mathcal{B} / \Gamma$ finite. From the convergence property, we see easily that for all $\epsilon>0$, the set $\{A \in \mathcal{B} \mid \lambda(A) \geq \epsilon\}$ is finite. Also, $\{A \in \mathcal{B} \mid \mu(A) \geq \epsilon, \lambda(A)>0\}$ is finite.

Now, given a symmetric annulus system, $\mathcal{A}$, and closed sets, $K, L \subseteq M$, we define, as in Section 6, $(K \mid L)$ to be the maximum number of nested annuli separating $K$ from $L$. (We will write $(K \mid L)_{\mathcal{A}}$ if there is any ambiguity.) In Section 6 , we 
introduced axioms (A1) and (A2) which together imply that (.....) is a hyperbolic path crossratio (Proposition 6.5). Here, we also introduce a weaker form of axiom (A3), namely:

(A4): If $x, y \in M$ are distinct, then $(x \mid y)>0$.

The proof of Theorem 8.1 will be based on the following general result:

Proposition 8.2. Suppose a group $\Gamma$ acts as a convergence group on a perfect metrisable compactum, $M$. Then, there exists a symmetric $\Gamma$-invariant annulus system, $\mathcal{A}$, such that if $(x, y, z, w) \in \Theta_{4}(M)$, then the three quantities $(x y \mid z w)$, $(x z \mid y w)$ and $(x w \mid y z)$ are all finite and at least two of them are equal to 0. Moreover, if $x, y \in M$ are distinct, then $(x \mid y)>0$.

In other words, we can always construct an annulus system satisfying axioms (A1), (A2) with $k=0$, and (A4).

In the proof, we shall make use of the space, $\Pi=\Theta_{2}(M)$, of distinct pairs of $M$. This is locally compact and metrisable. We fix some metric, $d$, on $M$. Given $n \in \mathbb{N}$, set $\Pi(n)=\{(x, y) \in \Pi \mid d(x, y) \geq 1 / n\}$. Thus, $(\Pi(n))_{n \in \mathbb{N}}$ gives a compact exhaustion of $\Pi$.

Proof of Proposition 8.2. We construct, inductively, a sequence of symmetric $\Gamma$ invariant annulus systems $\mathcal{A}(n)$ with $\mathcal{A}(n) / \Gamma$ finite. Writing $(K \mid L)_{n}=(K \mid L)_{\mathcal{A}(n)}$, we shall assume, inductively, that for all $(x, y, z, w) \in \Theta_{4}(M)$, at least two of the quantities $(x y \mid z w)_{n},(x z \mid y w)_{n}$ and $(x w \mid y z)_{n}$ are equal to 0 , and that for all $(x, y) \in \Pi(n)$ we have $(x \mid y)_{n}>0$. We can also assume that $\lambda(A)>0$ for all $A \in \mathcal{A}(n)$.

Suppose we have achieved this for a given $n$. Let $\mu$ be the minimal value of $\max \{\mu(\gamma A) \mid \gamma \in \Gamma\}$, as $A$ ranges over $\mathcal{A}(n)$. Thus, $\mu>0$. Now, given $\pi=(x, y) \in$ $\Pi(n+1)$, we choose an annulus, $A(\pi)=\left(A^{-}(\pi), A^{+}(\pi)\right)$, with $x<A(\pi)<y$, and with $A^{-}(\pi)$ and $A^{+}(\pi)$ sufficiently small such that $\mu(A(\pi))>1 /(n+2)$ (noting that $d(x, y)>1 /(n+1))$, and so that $\lambda(\gamma A(\pi))<\min \{\mu, 1 /(n+2)\}$ for all $\gamma \in \Gamma$ (noting that, from the previous discussion, we only need to consider finitely many $\gamma)$. We now choose a finite set, $\left\{\pi_{1}, \ldots, \pi_{p}\right\} \subseteq \Pi(n+1)$, so that $\Pi(n+1) \subseteq$ $\bigcup_{i=1}^{p}\left(\operatorname{int} A^{-}\left(\pi_{i}\right) \times \operatorname{int} A^{+}\left(\pi_{i}\right)\right)$. Let $\mathcal{B}=\bigcup\left\{\gamma A\left(\pi_{i}\right),-\gamma A\left(\pi_{i}\right) \mid 1 \leq i \leq p, \gamma \in \Gamma\right\}$. From the construction, we have $\lambda(A)<\min \{\mu, 1 /(n+2)\}$ for all $A \in \mathcal{B}$. Let $\mathcal{A}(n+1)=\mathcal{A}(n) \cup \mathcal{B}$. Again by construction, we have $(x \mid y)_{n+1}>0$ for all $(x, y) \in$ $\Pi(n+1)$.

Suppose, for contradiction, that there is some $(x, y, z, w) \in \Theta_{4}(M)$ with $(x y \mid z w)_{n+1}>0$ and $(x z \mid y w)_{n+1}>0$. In other words, there are annuli $A, B \in$ $\mathcal{A}(n+1)$ with $\{x, y\}<A<\{z, w\}$ and $\{x, z\}<B<\{y, w\}$. From the induction hypothesis, these cannot both lie in $\mathcal{A}(n)$, so without loss of generality, we have $A \in \mathcal{B}$. Moreover, after translating by some element of $\Gamma$, we can suppose that $\mu(B)$ is maximal in the $\Gamma$-orbit of $B$. It follows that $\mu(B)>\min \{\mu, 1 /(n+2)\}$ (depending on whether $B \in \mathcal{A}(n)$ or $B \in \mathcal{B})$. Since $A \in \mathcal{B}$, we see that $\lambda(A)<\mu(B)$. Thus, without loss of generality, we have $d(x, y) \leq \lambda(A)<\mu(B)$, contradicting the fact that $x<B<y$. We have verified the induction hypothesis for $n+1$.

Thus, starting with $\mathcal{A}(0)=\emptyset$, the induction proceeds for all $n$. Let $\mathcal{A}=$ $\bigcup_{n=1}^{\infty} \mathcal{A}(n)$, and write $(K \mid L)=(K \mid L)_{\mathcal{A}}$. Since $\Pi=\bigcup_{n=1}^{\infty} \Pi(n)$, we see that $(x \mid y)>0$ for all $(x, y) \in \Pi$.

It remains to verify that if $(x, y, z, w) \in \Theta_{4}(M)$, then $(x y \mid z w)<\infty$. Let $\lambda=$ $\min \{d(x, y), d(z, w)\}$, and choose some $n \in \mathbb{N}$ with $n>1 / \lambda$. We see that if $\{x, y\}<$ 
$A<\{z, w\}$, then $\lambda(A) \geq \lambda>1 / n$ and so $A \in \mathcal{A}(n)$. Since $\mathcal{A}(n) / \Gamma$ is finite, Lemma 7.2 tells us that there are only finitely many such annuli. The result now follows.

Lemma 8.3. Suppose that $\Gamma$ acts as a convergence group on $M$ and that $\mathcal{A}$ is a symmetric $\Gamma$-invariant annulus system satisfying (A4) above. If $x \in M$ is a conical limit point, and $K \subseteq M \backslash\{x\}$ is compact, then $(K \mid x)=\infty$.

Proof. Let $\left(\gamma_{i}\right), b$ and $c$ be as in the definition of a conical limit point. Now, $b \neq c$, so, by axiom (A4), there is some $A \in \mathcal{A}$ with $c<A<b$. Now, $\gamma_{i} \mid M \backslash\{x\}$ can be assumed to converge locally uniformly to $c$. Thus, for large enough $i$, we have $\gamma_{i} K<A<\gamma_{i} x$, and so $K<\gamma_{i}^{-1} A<x$. Thus, $(K \mid x)>0$, and so, by the inductive process described at the end of Section 6 , we see that, in fact, $(K \mid x)=\infty$.

Thus, if every point of $M$ is a conical limit point, we see that for any symmetric $\Gamma$-invariant annulus system, axiom (A4) implies axiom (A3).

Suppose now that $\Gamma$ satisfies the hypotheses of Theorem 8.1. Let $\mathcal{A}$ be the annulus system given by Proposition 8.2. We see that $\mathcal{A}$ satisfies axioms (A1), (A2) and (A3). Thus, by Proposition 6.5 and Lemma 6.6, we deduce:

Lemma 8.4. (.....) is a hyperbolic path crossratio on $M$, compatible with the given topology on $M$.

The constructions of Section 4 now give us a $\Gamma$-invariant path quasimetric, $\rho$, on $Q=\Theta_{3}(M)$, such that the boundary, $\partial Q$, is naturally identified with $M$ in such a way that the crossratios (..|..) and (..|..) $\rho$ agree up to at most an additive constant. Moreover, if $x, y, z \in M$ are distinct, then $(x, y, z) \in Q$ is a centre of the points $x, y, z$.

If we fix some $\theta \in \Theta_{3}(M)$, we get a map $f: \Gamma \longrightarrow Q$ defined by $f(\gamma)=\gamma \theta$. By Lemma 7.7, the pullback quasimetric $(\Gamma, \rho)$ is locally finite.

It remains to show that the $\Gamma$-orbit of a point in $Q$ is quasidense. However, we will need a different argument from Lemma 7.8.

Before we embark on this, we should clarify a few points arising from the dual role of $\Theta_{3}(M)$ as the locally compact topological space featuring in the hypothesis, and as the set (we have denoted by $Q$ ) on which we have defined our hyperbolic quasimetric.

Note that the space $\Theta_{3}(M)$ can be naturally compactified by adjoining a copy of $M$. This can be described by regarding $\Theta_{3}(M) \cup M$ as a quotient of $M \times M \times M$, where the quotient map is the identity on $\Theta_{3}(M)$, and sends a triple $(x, y, z)$ to $a \in M$ if at least two of the entries $x, y, z$ are equal to $a$. From this description, we see that if $\left(\left(x_{i}, y_{i}, z_{i}\right)\right)_{i}$ is a sequence in $\Theta_{3}(M)$, with $x_{i} \rightarrow a$ and $y_{i} \rightarrow a$ in $M$, then $\left(x_{i}, y_{i}, z_{i}\right) \rightarrow a$ in $\Theta_{3}(M) \cup M$. Moreover, the converse holds provided we allow ourselves to permute the entries of a triple.

Now, suppose $\rho$ is a hyperbolic path quasimetric on $Q=\Theta_{3}(M)$ (such as that which we have constructed) with the property that $\partial Q$ is identified with $M$ in such a way that for all distinct $x, y, z \in M \equiv \partial Q$, the point $(x, y, z) \in Q$ is a centre of the triple of ideal points, $x, y, z$, with respect to the metric $\rho$. Now, as discussed in Section 3, $\Theta_{3}(M) \cup M \equiv Q \cup \partial Q$ admits a geometrically defined topology (where we view $Q$ as discrete). From the earlier description of $\Theta_{3}(M) \cup M$, we see that a sequence, $\left(u_{i}\right)_{i}$, of distinct triples, converges in the (compact) topology on $\Theta_{3}(M) \cup M$ if and only if it does so in the geometric topology. We can thus speak unambiguously of such a sequence converging. One consequence is the following. 
Suppose $R \subseteq Q \equiv \Theta_{3}(M)$. If we say that $R$ is "relatively compact", we mean that its closure in $\Theta_{3}(M)$ is compact (or equivalently that its closure in $\Theta_{3}(M) \cup M$ does not meet $M$ ). If we say that it is "bounded", we mean that it has finite diameter in the quasimetric $\rho$. In fact, the notions are equivalent. This is based on the following two observations. Firstly, any sequence in $\Theta_{3}(M)$ converging to a point of $M$ is unbounded. Secondly, any unbounded sequence in $\Theta_{3}(M)$ has a subsequence which converges to a point of $M$. Both these statements follow by simple geometric arguments, given our earlier discussion of convergence.

We now return to the question of showing that, in our particular situation, the orbit of a point is quasidense. There is a fairly standard argument for hyperbolic path metric spaces, which we can push through in this more general situation, given the observation made above that a subset of $\Theta_{3}(M)$ is relatively compact if and only if it is bounded.

Lemma 8.5. If $\theta \in Q$, then the orbit $\Gamma \theta$ is quasidense in $Q$.

Proof. Suppose not. Then there is a sequence, $\left(u_{i}\right)_{i}$, in $Q$ with $\rho\left(u_{i}, \Gamma \theta\right) \rightarrow \infty$. Since $\rho$ is $\Gamma$-invariant, we can suppose that $\theta$ is a nearest point of $\Gamma \theta$ to $u_{i}$ for all $i$. Now, $\left(u_{i}\right)_{i}$ is unbounded, and so, after passing to a subsequence, it can be assumed to converge on some point $x \in M$ (see the earlier discussion). If $\left(v_{i}\right)_{i}$ is another sequence in $Q$ converging to $x$, and remaining a bounded distance from some geodesic ray, then a simple geometric argument shows that $\rho\left(v_{i}, \Gamma \theta\right) \rightarrow \infty$.

By hypothesis, $x$ is a conical limit point. Thus, we can find a compact set, $\Theta_{0} \subseteq \Theta_{3}(M)$, a point $y \in M \backslash\{x\}$, and a sequence $x_{i} \rightarrow x$ in $M$, such that $v_{i}=\left(y, x, x_{i}\right) \in \bigcup \Gamma \Theta_{0}$ for all $i$. But now, $\Theta_{0}$ is bounded, and so $\rho\left(v_{i}, \Gamma \theta\right)$ is bounded. However, the points $v_{i}$ all lie a bounded distance from any bi-infinite geodesic connecting $y$ to $x$, contradicting an earlier statement.

We now see that $\Gamma$ is hyperbolic as before. In fact, we have a bounded subset, $R \subseteq Q$, with $Q=\bigcup \Gamma R$. Let $\Theta_{0}$ be the closure of $R$ in $\Theta_{3}(M)$. It follows that $\Theta_{0}$ is compact. Now, $\Theta_{3}(M)=\bigcup \Gamma \Theta_{0}$, showing that $\Gamma$ is a uniform convergence group. This proves Theorem 8.1.

\section{REFERENCES}

[BeM] A.F.Beardon, A.Maskit, Limit points of kleinian groups and finite sided fundamental polyhedra, Acta Math. 132 (1974) 1-12. MR 48:11489

[Bo1] B.H.Bowditch, Notes on Gromov's hyperbolicity criterion for path-metric spaces, in "Group theory from a geometrical viewpoint" (ed. E.Ghys, A.Haefliger, A.Verjovsky), World Scientific (1991) 64-167. MR 93h:57002

[Bo2] B.H.Bowditch, Geometrical finiteness with variable negative curvature, Duke Math. J. 77 (1995) 229-274. MR 96b:53056

[Bo3] B.H.Bowditch, Cut points and canonical splittings of hyperbolic groups, to appear in Acta. Math.

[Bo4] B.H.Bowditch, Convergence groups and configuration spaces, to appear in "Group Theory Down Under" (ed. J.Cossey, C.F.Miller, W.D.Neumann, M.Shapiro), de Gruyter.

[Bo5] B.H.Bowditch, Relatively hyperbolic groups, preprint, Southampton (1997).

[CanS] J.W.Cannon, E.L.Swenson, Recognizing constant curvature discrete groups in dimension 3, to appear in Trans. Amer. Math. Soc. CMP 97:15

[CasJ] A.Casson, D.Jungreis, Convergence groups and Seifert fibered 3-manifolds, Invent. Math. 118 (1994) 441-456. MR 96f:57011

[D] M.J.Dunwoody, The accessibility of finitely presented groups, Invent. Math. 81 (1985) 449-457. MR 87d:20037

[F] E.M.Freden, Negatively curved groups have the convergence property, Ann. Acad. Sci. Fenn. Ser. A Math. 20 (1995) 333-348. MR 96g:20054 
[Ga] D.Gabai, Convergence groups are fuchsian groups, Ann. of Math. 136 (1992) 447-510. MR 93m:20065

[GeM1] F.W.Gehring, G.J.Martin, Discrete quasiconformal groups I, Proc. London Math. Soc. 55 (1987) 331-358. MR 88m:30057

[GeM2] F.W.Gehring, G.J.Martin, Discrete quasiconformal groups II, handwritten notes.

[GhH] E.Ghys, P.de la Harpe (eds.), Sur les groupes hyperboliques d'après Mikhael Gromov, Progress in Maths. 83, Birkhäuser (1990). MR 92f:53050

[Gr] M.Gromov, Hyperbolic groups, in "Essays in Group Theory" (ed. S.M.Gersten) M.S.R.I. Publications No. 8, Springer-Verlag (1987) 75-263. MR 89e:20070

[HK] J.Heinonen, P.Koskela, Quasiconformal maps in metric spaces with controlled geometry, to appear in Acta. Math.

[K] R.Kirby (ed.), Problems in low-dimensional topology, problem list, Berkeley (1995). CMP 98:01

[MT] G.J.Martin, P.Tukia, Convergence groups with an invariant component pair, Amer. J. Math. 114 (1992) 1049-1077. MR 93i:30034

[O] J.-P.Otal, Sur la géométrie symplectique de l'espace des géodésiques d'une variété à courbure negative, Rev. Math. Iberoamericana 8 (1992) 441-456. MR 94a:58077

[P] F.Paulin, Un groupe hyperbolique est determiné par son bord, J. London Math. Soc. 54 (1996) 50-74. MR 97d:20042

[T1] P.Tukia, Homeomorphic conjugates of fuchsian groups, J. reine angew. Math. 391 (1988) 1-54. MR 89m:30047

[T2] P.Tukia, Convergence groups and Gromov's metric hyperbolic spaces, New Zealand J. Math. 23 (1994) 157-187. MR 96c:30042

[T3] P.Tukia, Conical limit points and uniform convergence groups, preprint, Helsinki (1996).

Faculty of Mathematical Studies, University of Southampton, Highfield, Southampton SO17 1BJ, Great Britain

E-mail address: bhb@maths.soton.ac.uk 